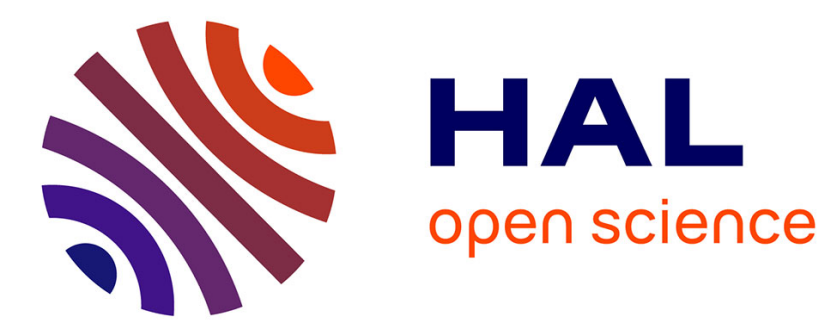

\title{
Topographie antique de Marseille: le théâtre et le mur de Crinas
}

\author{
Fernand Benoit
}

\section{To cite this version:}

Fernand Benoit. Topographie antique de Marseille: le théâtre et le mur de Crinas. Gallia - Fouilles et monuments archéologiques en France métropolitaine, 1966, 24 (1), pp.1-20. 10.3406/galia.1966.2435 . hal-01934386

\section{HAL Id: hal-01934386 https://hal.science/hal-01934386}

Submitted on 26 Feb 2020

HAL is a multi-disciplinary open access archive for the deposit and dissemination of scientific research documents, whether they are published or not. The documents may come from teaching and research institutions in France or abroad, or from public or private research centers.
L'archive ouverte pluridisciplinaire HAL, est destinée au dépôt et à la diffusion de documents scientifiques de niveau recherche, publiés ou non, émanant des établissements d'enseignement et de recherche français ou étrangers, des laboratoires publics ou privés.

\section{(이) $\$$}

Distributed under a Creative Commons Attribution - NonCommercial - NoDerivatives| 4.0 


\title{
TOPOGRAPHIE ANTIQUE DE MARSEILLE : LE THÉÂTRE ET LE MUR DE CRINAS
}

\author{
par Fernand BENOIT
}

\author{
TIEATTRE
}

Les vestiges découverts en 1946-1948 au théâtre. dont les grarlins sont adossés à la butte Saint-Laurent (ils avaient été recouverts par mesure de protection au cours des travaux de reconstruction du quartier), ont été remis au jour en 1963, dans le square compris entre les deux ailes d'un groupe scolaire, rue des Vartégales (Inv. Fouilles III).

Il ne subsiste que deux éléments des grarlins inférieurs (fig. 1 et 2 ) de la cavea, protégés par l'angle mort de la butte et une portion du sol bétonné de l'orchestre conservée sous la rue Perdigone, entre les raves des maisons ${ }^{1}$; aucune trace des parodoi, entrées latérales, ni des substructions du mur du proscirnium, qui limitait l'orchestre. les caves des maisons dr l'ancienne rue des Martégales ayant fait disparaître le sol antique. La surveillance des travaux de construction du mur de soutènement de la butte Saint-Laurent (anciennes rues des Ferrats et du Château-Joly) n'a apporté aucun élément qui permît de reconnaitre les parties supéricures de la carea. qui devait s'élever à la cote $\pm 24 \mathrm{~N}$ (iF. correspondant a la terrasse supérieure de la butte du "Chàteau Babon ", houleversée par les caves et les ranalisations d'égout. Le niveau du béton de l'orchestre, pulvérulent et dont la rouche superficielle plus comparte avait disparu (figr. 13), peut être fixé par la présence d'une plaque de marbre blane de calage, incrustée dans la partie n.-o. de l'orchestre. Cie point est à la cote $+5 \mathrm{~m}$ du $\mathrm{X}(\mathrm{GF}$, soit à 10.21 sous le niveau du dallage de la pracinctio inférieure de la carea. Mais une tranchée, de $3.50 \mathrm{~m}-4 \mathrm{~m}$ environ de largeur, faite à l'époque d'abandon du théâtre, au we-ve siècle, d'après la datation du dépotoir² qui recouvrait le site. a fait disparaitre toute trace du canal recouvert de dalles, concentricue à l'orchestre, qui servait à l'écoulement des eaux de pluie.

l.a nature des terres d'apport qui formaient l'assise de l'orchestre a provoqué un affaissement général du sol, surtout sensible dans la partie s.-e. et qui avait entraîné le fléchissement des dalles de la prescinclio, qui ont été replacées à leur niveau. L'affaissement

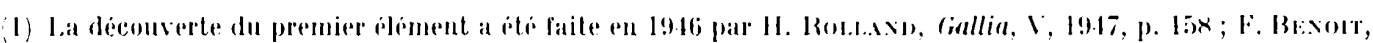
ibid., VI, 194x, p. 2117; XX, 1962, p. 687; cKA I, 1947, p. 5x:3.

2) Secteur de fouilles III : alondante céramique grise estampie, en partie publice par J. Lucom, La réramique palen-chretienne sigillee grise, $195 x$ topographie, p. 91). 


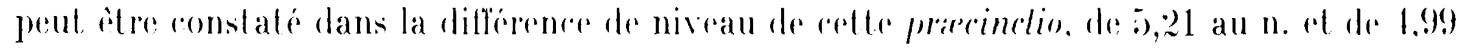
alls.

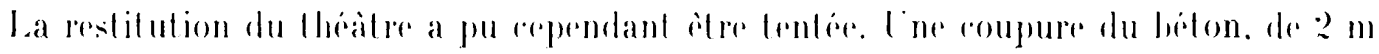
emviron de longueur, reconnue en 1918, dans la partir n.-0. de l'orehestre. preperndiculaire a l'alignement des caves de la rue Perdigone qui gravisiail la jente de la butte. el la ronca-

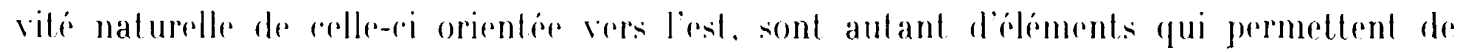

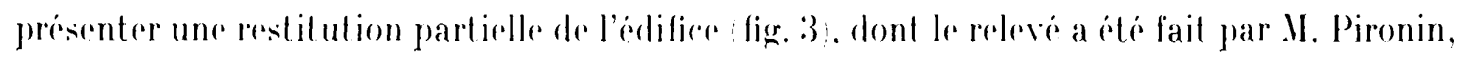
dessinaleur du Servier d'arehiterture antique du li.X.R.s. fier. 1). I.existence du beton, ou tout au moins de la surface lassée du lit de déchets de laille de pierre de la couronne. qui supportait le dallage de l'orehestre. au-rlela du diametre du demi-cerele de celui-ei, sur
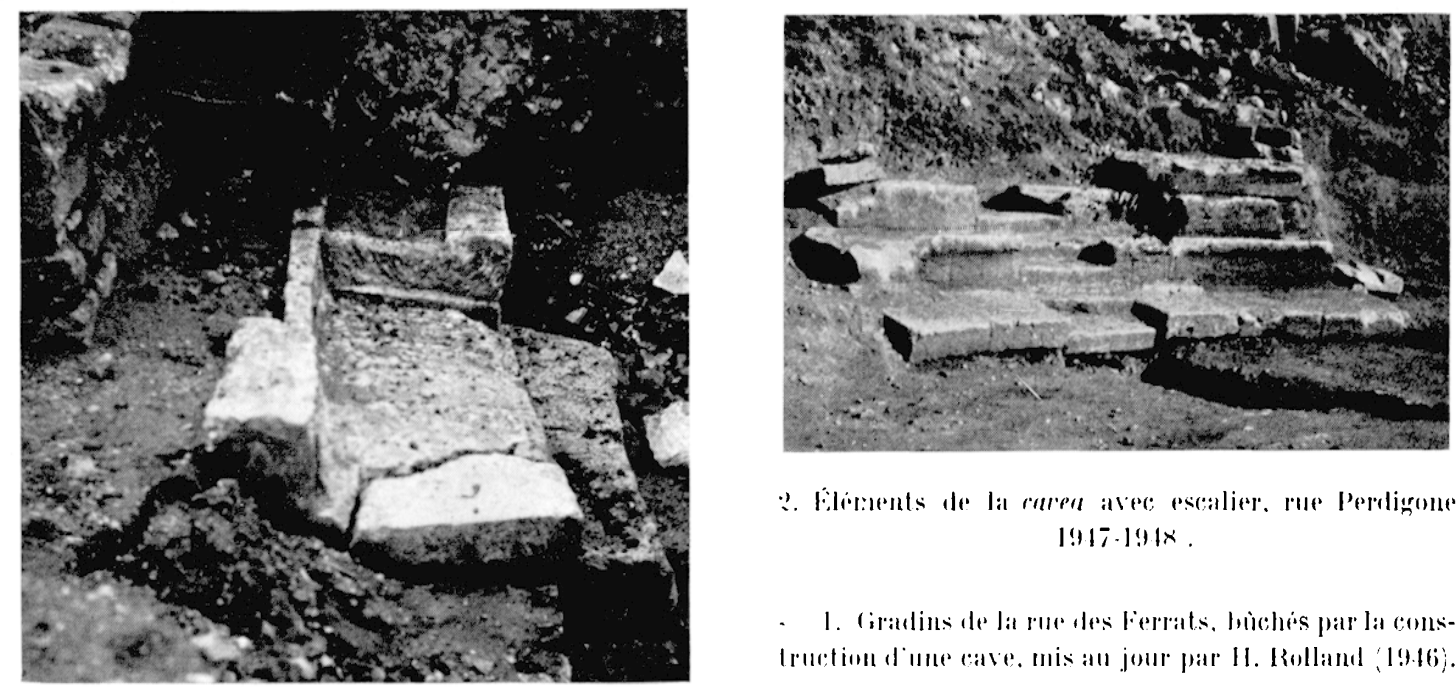

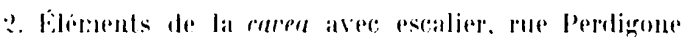
$1917 \cdot 1918$.

1. Comalins de la rue des lierrats, bioches par la coms-

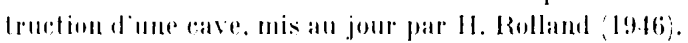

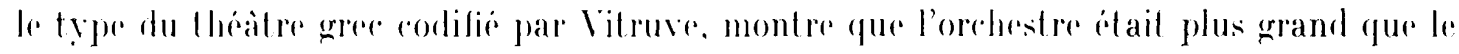

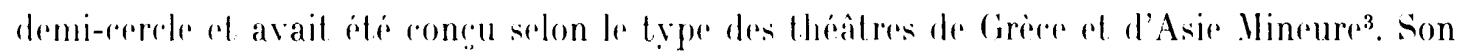

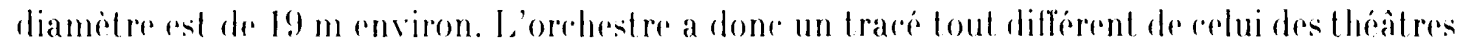
momains. d'Arles. de Frejus, d'(trange. de lëenne. construits sous Auguste. qui ne dépasse jas le diametre du cerele. II se rapproche du type des théatres hellenistiques de Ségeste ou de Pompeid, dont le demi-rerele de l'orehestre est prolonge par deux demi-rayons paralleles qui viennent buter contre le pulpilum du prescienium.

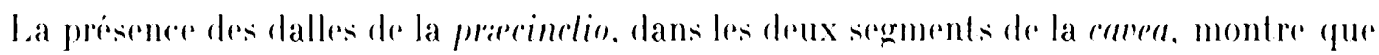
les gradins deserendaient jusqu'au niveau dre l'orehestre. sans suredevation d'un podium, qui est raracteristique des theitres-amphilhesters d'Asir Mineure Cetle structure est

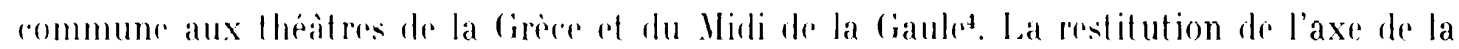

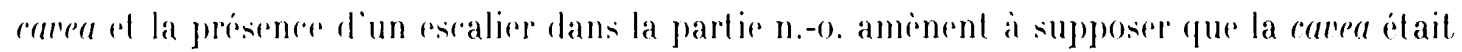
parlageren ring cunei par six escaliers repartis de part el d'autre de l'axe. selon le tyge du

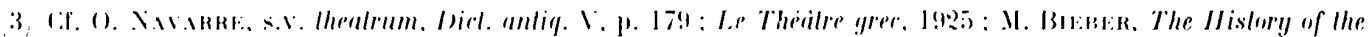

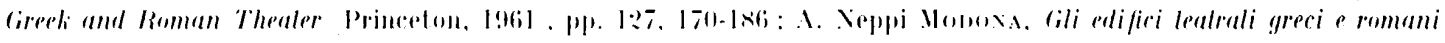
llorence, lafil,

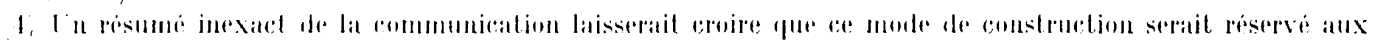

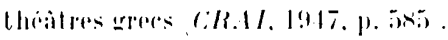




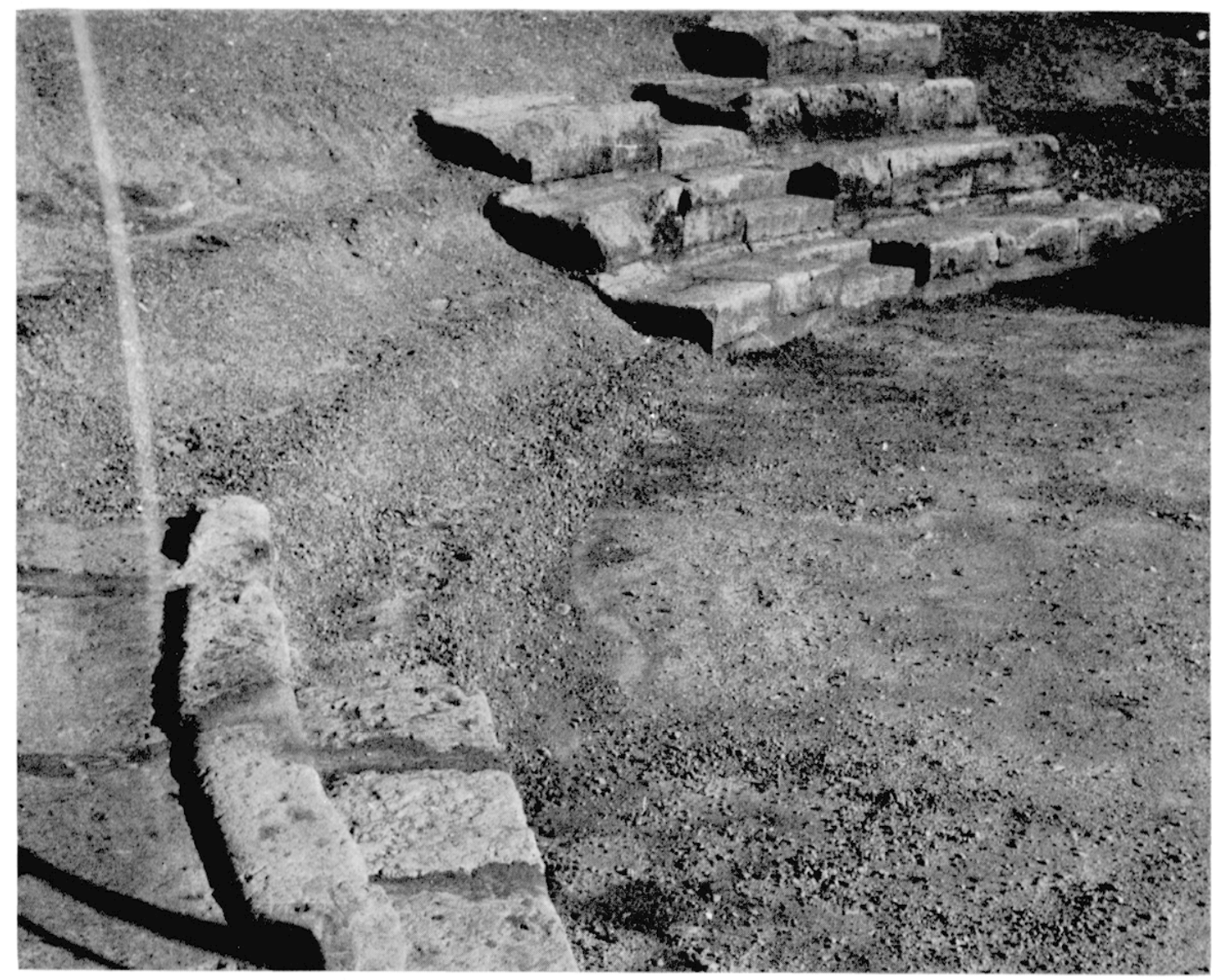

3. Les deux segments de la canea remis an jomr en lofi3.

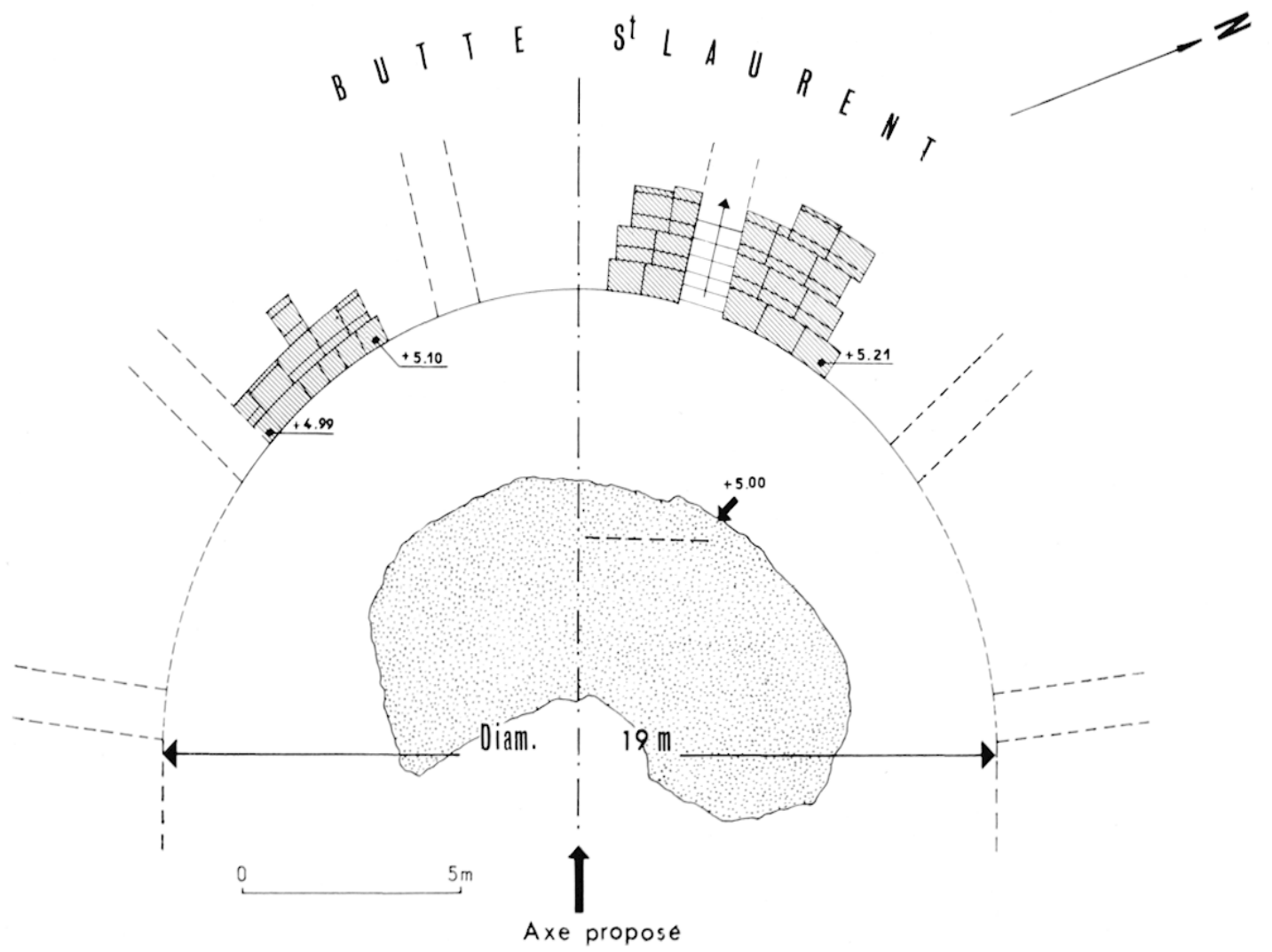

4. Projet de restitution du thatre, par P'. Pironin (1961). 
théàtre gree. L'acces des gradins supérieurs devait se faire par le haul. selon une disposition adoptée au théàtre de Vaison, également arlossé a une colline; mais l'existenere de romiloria, raractéristiques des théatres romains, parait être attestié par la découverte sur le remblai au n. des gradins, d'une grande dalle, chanfreinée, aver moulure diagonale (fig. 5), qui correspondrait au chambranle de l'un des accès sur une priccinclio.

Les similitudes de re plan aver celui du théatre gree. non moins que l'identité de profil des banquettes, inconnu dans les théatres romains de la Gaule, posaient un problème de rlatation qui ne pouvait être résolu que par des sondlages moins limités que rerlui qui avait été fait en 1948, sous le béton de l'orchestre. L'évaruation des ruines des maisons at la libération du chantier en 1963-64, après la construction du mur de soutenement de la butte et du groupe scolaire, ont rendu possible une série d'observations qui ont cete faites par des sondages sous le niveau de l'orchestre et sous te lit de pose des gradins ${ }^{5}$.

I. Sous-sol de la can'ea (fig. 6). ... Deux dalles (1 el 2) de la pracinclio inférieure et deux gradins (15 et 16). dont le parfait jointoiement prouvait qu'il n'avait pu y avoir d'infiltration sous leur assise. ont élé soulevés en rue de reconnaître le mode de const ruction et de prélever, éventuellement. des tessons de céramique qui puissent dater l'époque de la construction (fig. 7). Le graltage du lit de pose sous les dalles 1 el ?. qui étaient inclinées sur la tranchée faite à l'époque paléochrétienne pour récupérer les pierres de la canalisation découlement des eaux pluviales. n'a rien donné dans la partie oil elles reposaient sur le sol vierge; en bordure, quelques tessons romains el "wisigothiques" s'élaient insérés sous la partie antérieure de la dalle. Yous avons donc souleré les dalles 15) et 16 appartenant is la rangée supérieure : sous la dalle 16 . aucun tesson. ni aucune infiltration ayant pu provenir de la partie supérieure: la dalle 15, par contre. a permis d'éludier la technique do la pose (fig. 8 ) : l'emplacement des gradins avait été préparé par la taille de la rolline. selon le mode qui sera suivi à Vaison pour la construction du théatre. L'espace libre entre l'arrière du gradin de la rangée inférieure el le lit dallente du gradin 15) avail ele rempli avee de la raillasse et quelques déchets de taille, au milieu desquels ont été recueillis plusieurs tessons de poterie : panse d'amphore massalicte. fragment de vase en pate rougre al tribuable i l'époque romiaine. dont un parail avoir été roulé par les vagues et provenir par conséquent de terres d'apporl de la plage el deux fragments de vases indigines. en lerre noiritre micacée (indalables) : aucun morceau de poterie grecque n'a élé trouvé dans celte opération. Le sol vierge avait été damé el lissé a la truelle (lip-tapi de facon ì constiluer une assise d'altente parfaitement jointive avec le lit de pose de la dalle.

Le profil des gradins est de tradition grecque; mais reux-ci ne présentent aucune marque. aucun signe de pose. Au lieu d'itre parallélipipédiques comme ceux des théitres du Midi de la Gaule, qui ne sont pas antérieurs a luguste, ils présentent sur leur face supérieure un profil mouluré unique en Gaule : le siège forme en avant un léger encorbellement en relief qui détermine dans la partie arriere de la banquette une large goultiere permetlant au spectateur de replier les jambes, de facon is ne pas incommoder le spectateur de la file inférieure (fig. 9). I'n tel profil, courant en (irice dans les théitres, les amphithéatres et les stades, avait ćăalement até adoplé dans les théit res d'époque romaine de Pompéi et d'llippone.

L'appareillage a joints vifs est remarquable par sa perfection : les joints présentent sur la tranche un cadre ciselé (analhylrose) pour l'adhésion des blocs. selon la lechnique orrecque ${ }^{6}$. Cependant le relevé des profils montre des irrégularités qui révilent une époque lardive. Les gradins, d'une hauteur de 0.32 i 0.34 , ont une largeur differente. variant entre 0.72 .76 .81 et $\$ 2$; le lit

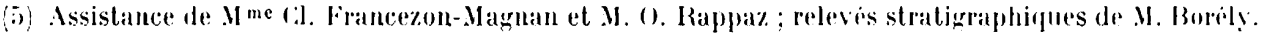

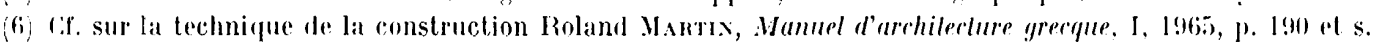




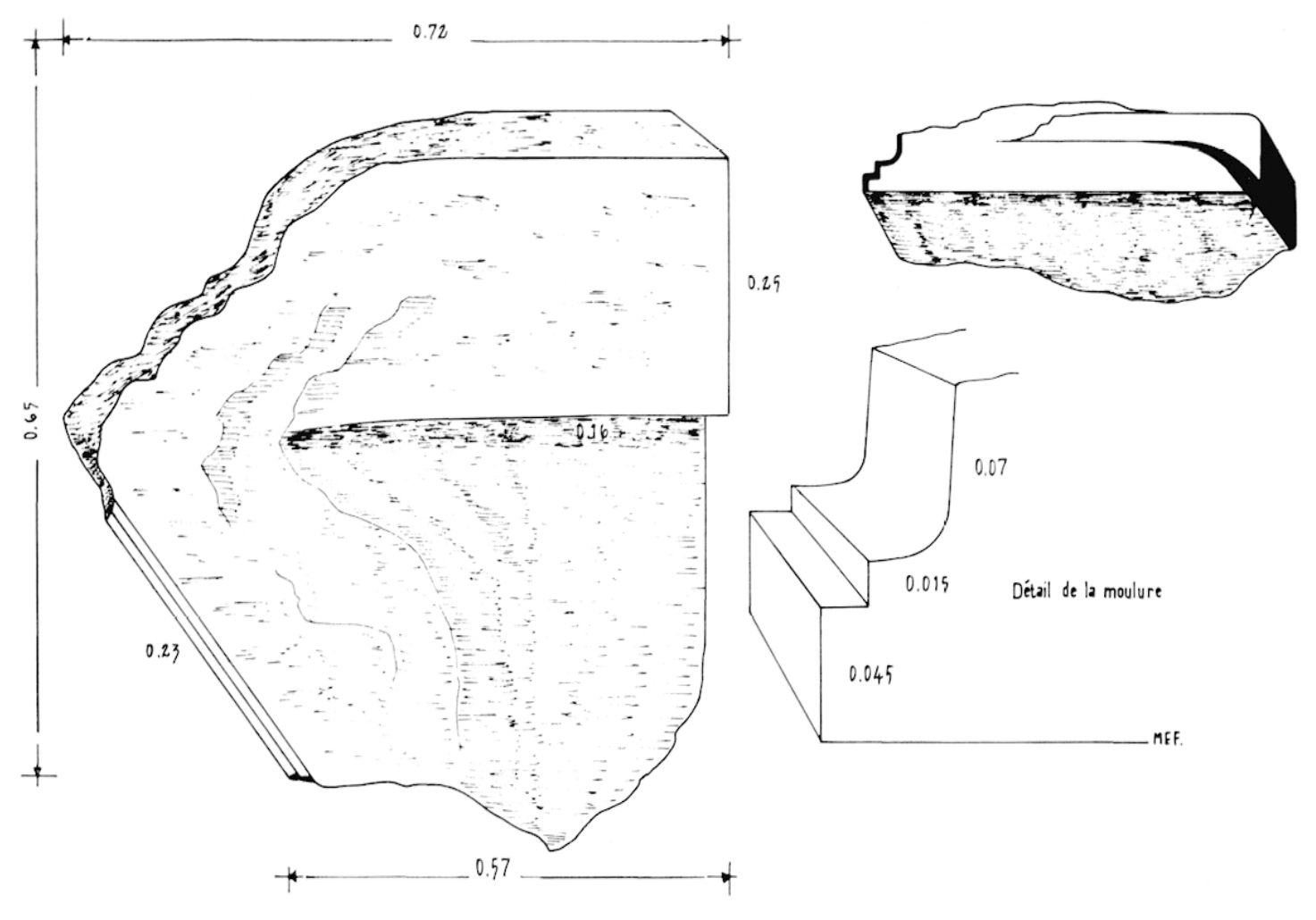

5. Dalle chanfreinie et moulure (cf. fig. 2).

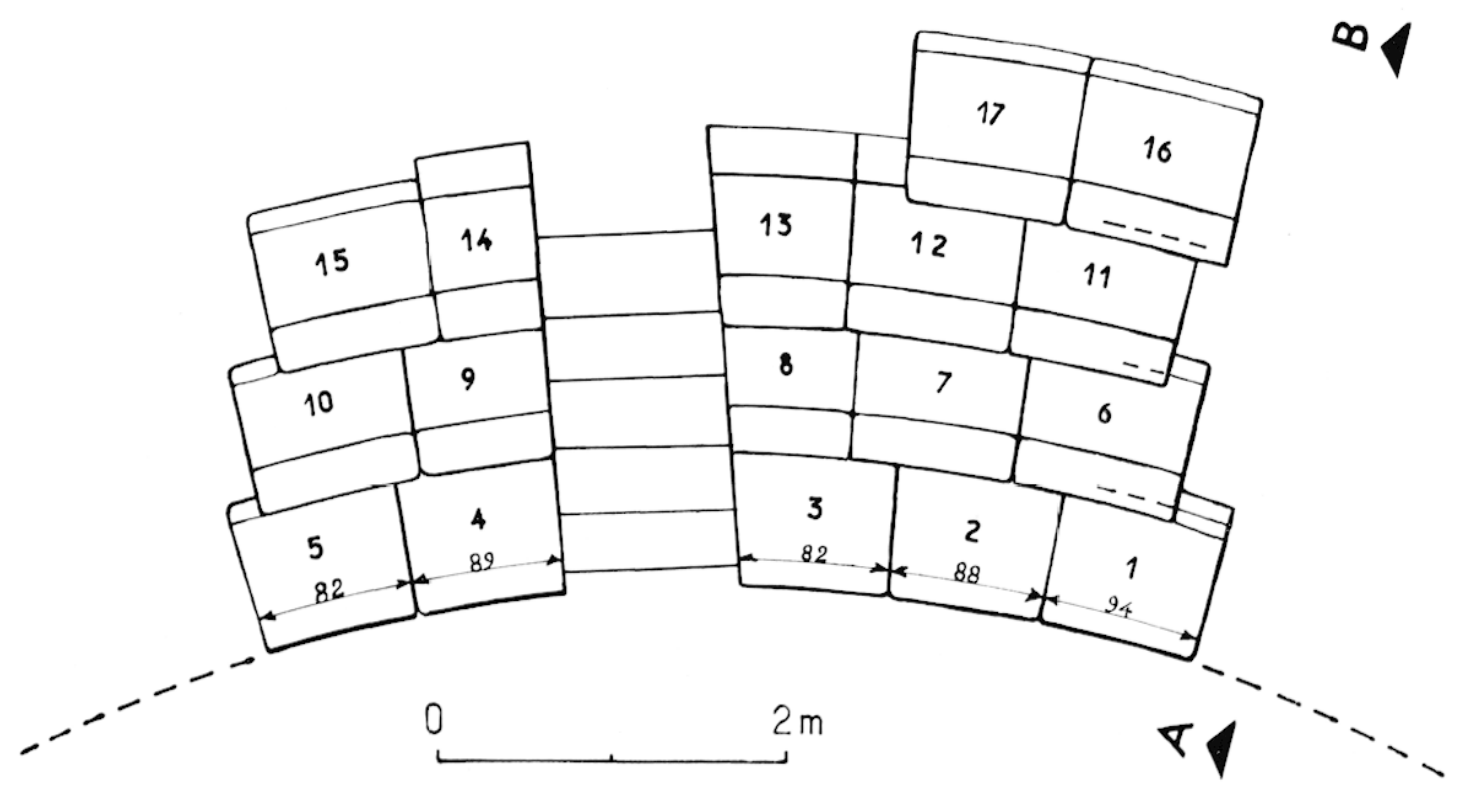

6. Plan des gradins et de l'escalier de l'élement nord. 


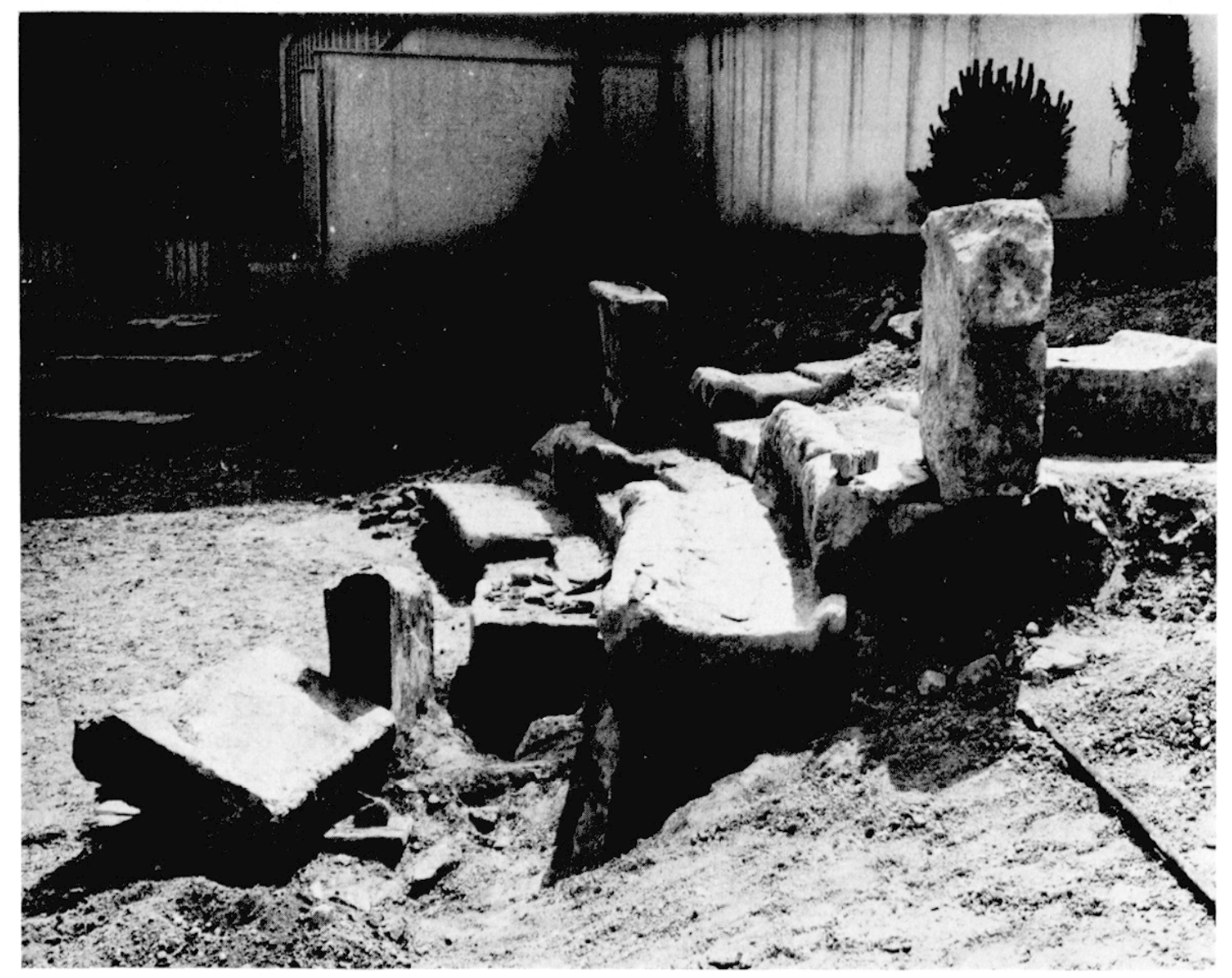

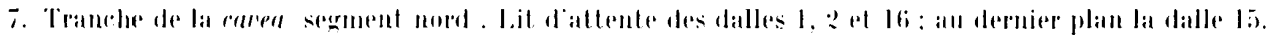

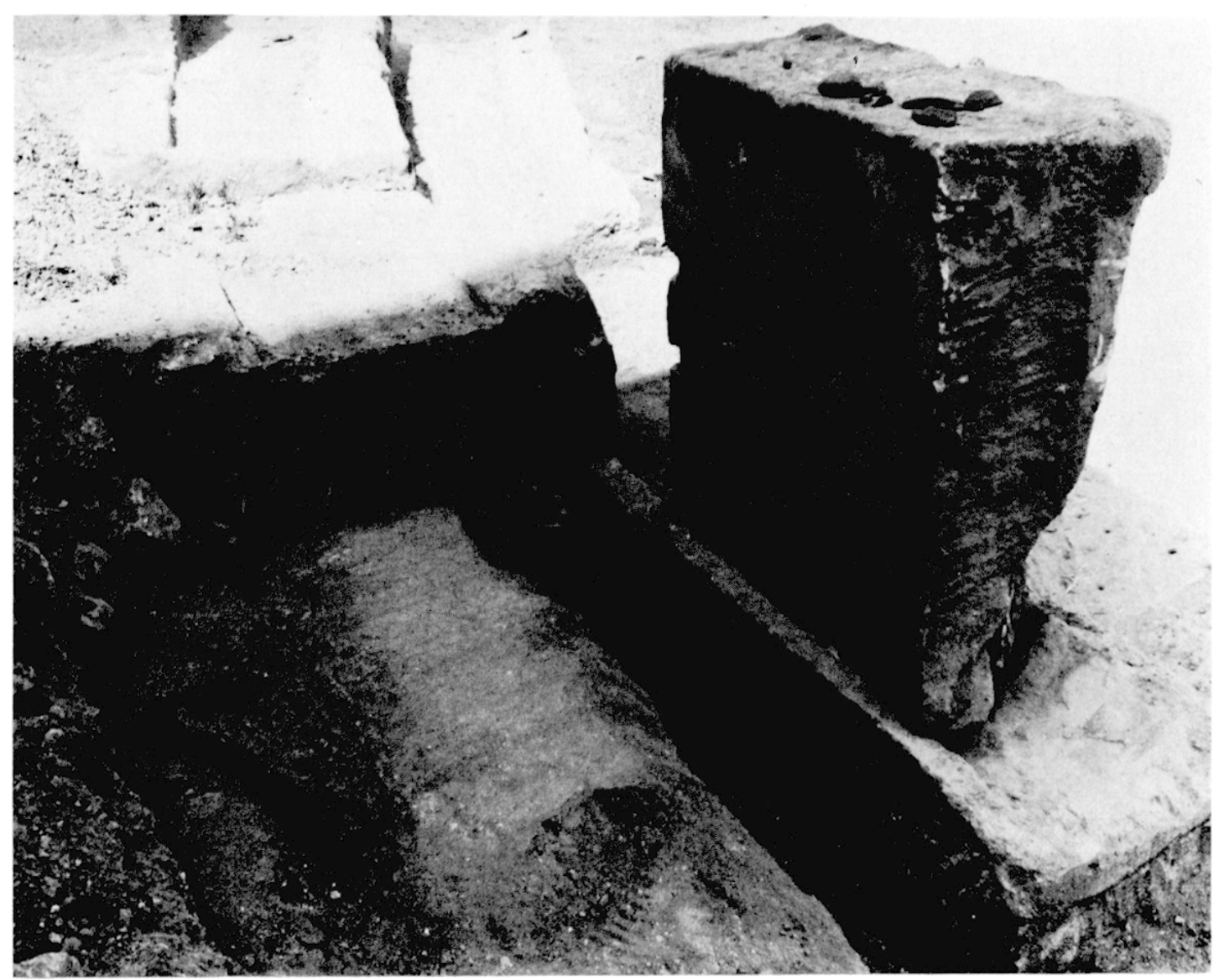

$x$. Lit diattente de la dalle lis. 


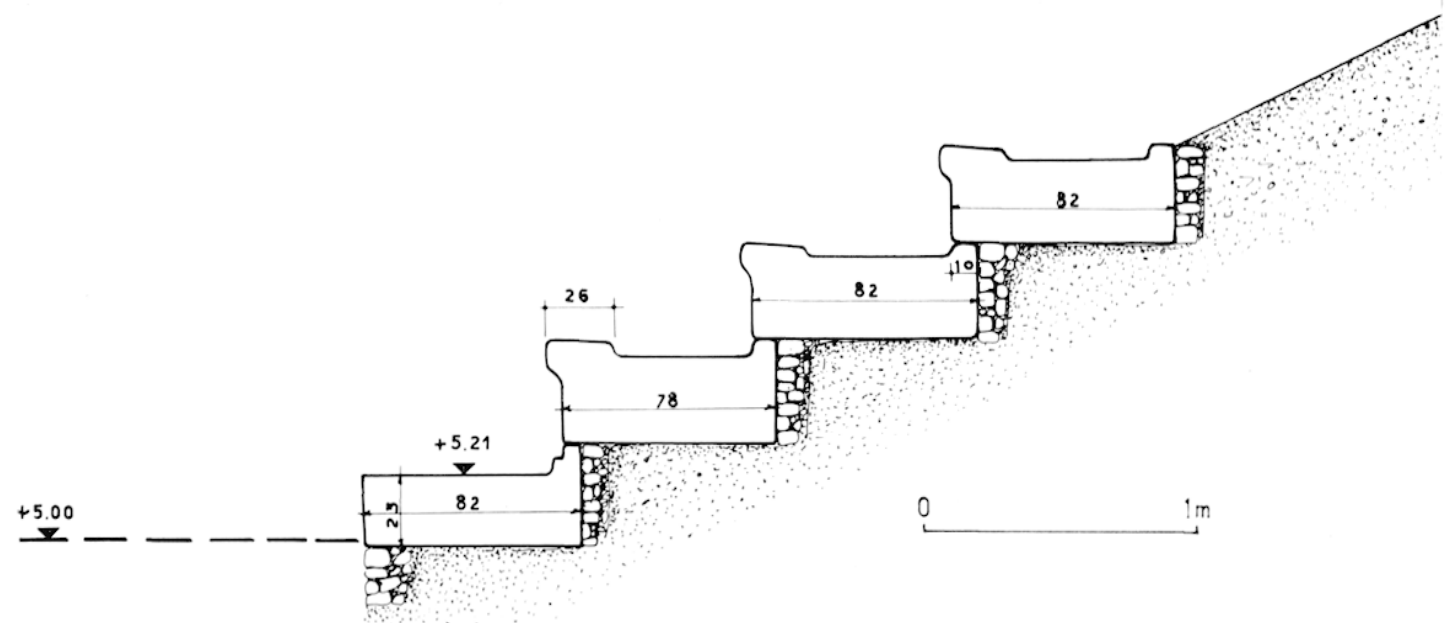

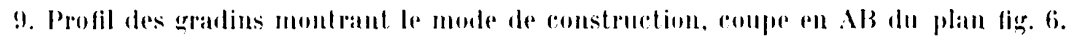

d'altente menage a larriere de chapue gradin pour recevoir le gradin superieur a une largeur variable. de 0.06 . a 0.09 ef mime 0.26 .

II. Orchestre. Les sondages faits en 19487, sous le beton de lorrhestre dans la partie n.-o., avaient révé que le lit supérieur était composé de déchets de taille de calcaire de la Couronne. liés a un mortier pulvérulent. aver quelques fragments de béton de luileau. Il ne subsistail qu'une petite tabletle de marbre blanc, qui avait servi au calage du dallage de marbre multicolore, dont il ne restail aucun vestige en place. mais dont ont été recueillis de tris nombreux fragments au cours du déblaiement.

Le niveau actuel de l'orchestre, dans celte partie, est a la cole + $\mathrm{m}$ du N(il. le sol s'élant allaisse de plus de $0,20 \mathrm{~m}$ dans sa partie sud. La coupe du sous-sol de l'orchestre montre que celui-ci se composait de lits alternés de déchets de taille de calcaire de la Couronne preure que les gradins araient éte laillés sur le chantier de construction de la rarea (fig. 10). Le bélon avail été entaillé, nous l'avons dit, par une profonde lanchee concentrique a la cale'a. Le comblement a domne une

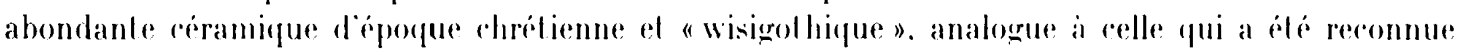
dans le dépotoir qui recouvrait le théal res.

1. La tranchée du nord (111. 4. 19.48: fig. 10. I) : poussée à 2,70 sous l'orehestre, a donne la stratigraphie suivanle. sans lesson de réramique :

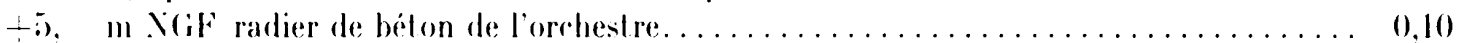

$.90 \mathrm{~m} \quad$ congloméral de déchets de taille el de cailloux $\ldots \ldots \ldots \ldots \ldots \ldots \ldots \ldots \ldots$ 0.40

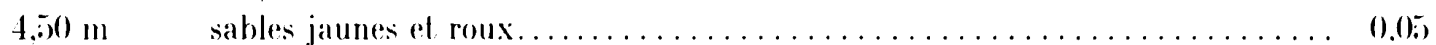

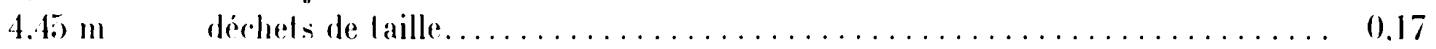

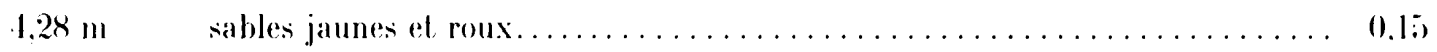

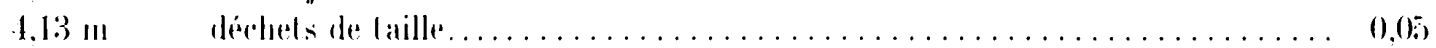

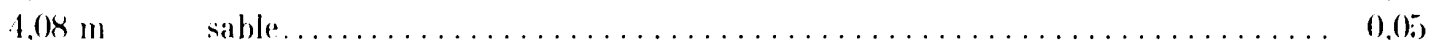

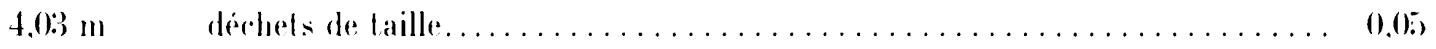

$3.98 \mathrm{~m}$ sable jaune. marin et pluvialile. apporte pour remplissage aver quelques. déchels de laille de calcaire de la Couronne................... 1.68

2.30 m marne el pourlingue. 


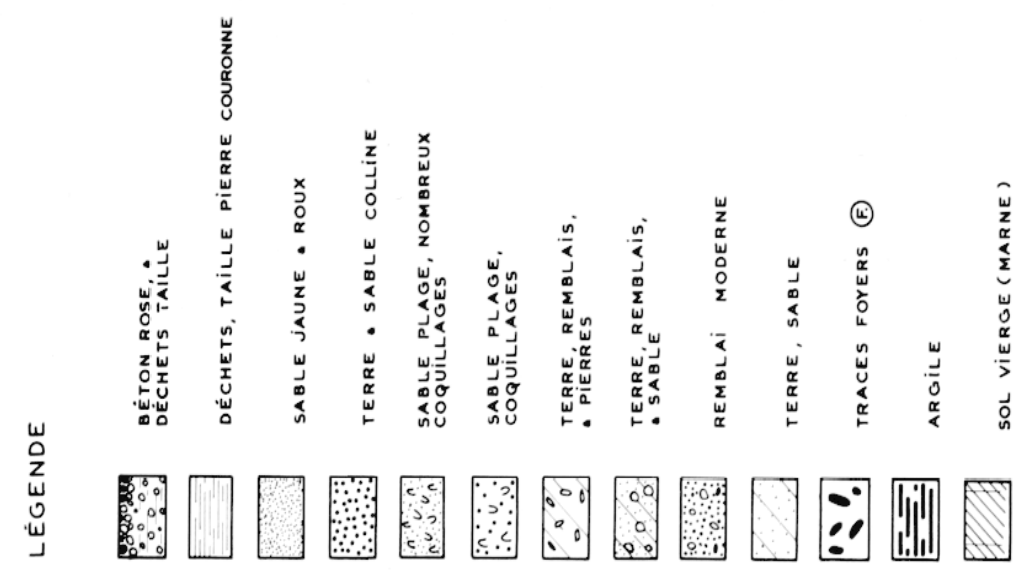

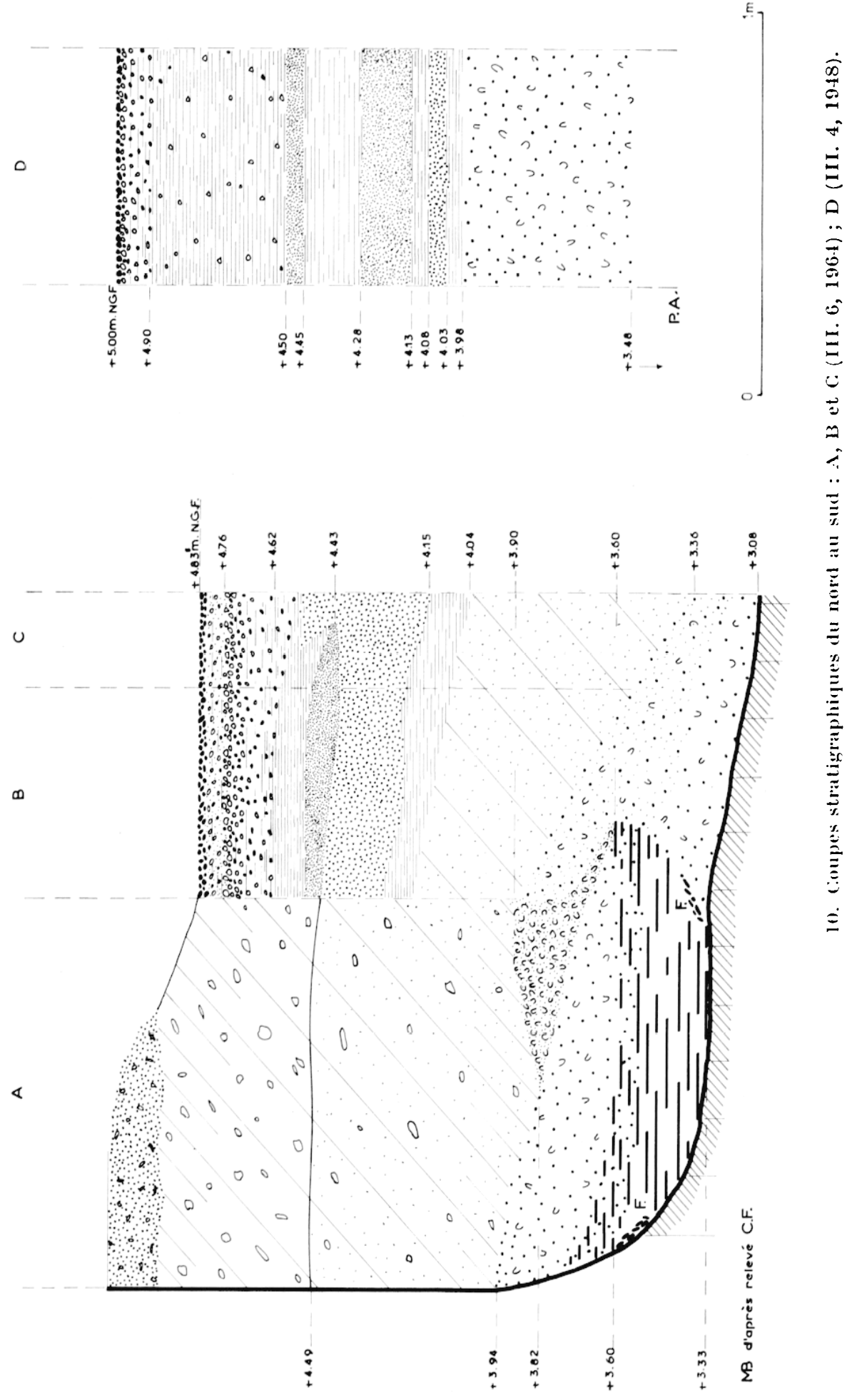


2. Tranchée III. 3. 19.tx. Ia mime formation a été reconnue par un sondage vers le centre de lorchestre. au droit de la coupure du beton ; la coupe de terrain a montré un sol profondément remanié à l'époque paléochrétienne : ont été recueillis des fragments ionien el rampanien, associés a des fragments de polerie sigrillée et de lampes chrétiennes. Le sondage a élé poussé en lunnel sous le béton de l'orchestre. de maniere a lrouver une stratigraphie en place. la composition du sous-sol, aver alternance de déchets de taille el de sables, est identique a celle qui avait éte déterminée au poinl précédent.

De la cote 3.50 a 2.70 au-dessus du N(il onl été trouvés:

un petil fragment de pied de coupe allique, des fragments de grands vases en lerre jaune clair trés micacée, donl un fragment damphore a bouton massaliete : un fragment de luile a crochet. en terre jaune micacée (en "écaille de poisson "). du type analogue aux tuiles romaines des fours de Saint-llenry ${ }^{9}$; deux fragments de vases de couleur ocre rouge i cercles brunitres et a engobe blanchatre; un fragment céramique avec coquillage marin adhérent.

[ $)+2.70$ a -2.50$)$. la coupe du terrain a fait apparaître un comblement par des sables marins mélangés à des éléments oligocines de la colline, avec railloux, débris durgonien el bois carbonisés. Les éléments marins comprennent. a l'exception d'un chama. coquillare d'eau vive, une faunule d'eau saumatre abondante sur la plage. caractéristique du lacydon a l'époque hellénistique et romaine, oì domine le cardium edule, ayant encore ses deux valves (loripes lacleus, gibbula Adansoni, calliosloma striala) : celte faunule est mélangée a des déchets de cuisine (oslraea, lapes aureus el decussalus etc. ${ }^{10}$.

Le, 2,50 a 2,30, le comblement de sable marin el de débris d'urgonien se poursuit avec coquillages d'eau saumaitre el huitres et quelques fragments de poterie micacée massalipte de polerie rouge roule par l'eau. un lesson de coupe altique a vernis noir viré au rouge et une plaque de marbre de Carrare en trois fragments (0,15), 0,13, 0.00): : III. 3. couche 1), 52). corrodés par les infiltrations d'eau salée ${ }^{11}$. dont l'origine ne peul itre antérieure à l'Empire el la provenance indique un abandon sur la plage du lacydon à l'époque romaine.

3. In nouveau sondage (III. (6, 1964: fig. 10, A, B, C) a été fait a la limite s. du béton de l'orchestre: la coupe du terrain a permis de faire une stratigraphie en tranchée jusqu'au sol vierge a 3,08 du $\mathrm{N}(\mathrm{iF}$, correspondant au niveau $+2,30$ mis au jour au n.-o., preuve que le sol de la calanque était inégal el avait élé comblé jusqu'i la cote +5 pour asseoir l'orchestre du théitre. la fouille a dù déblayer une importante couche de terres d'apport (lig. 10 A), dans lesquelles ont elé lrouves des tessons de l’̈poque qrecque a l’époque barbare : fragments d'amphores romaines (anses el panses). fragments de céramique grise estampée du Iv-ve s., dont deux a dessin inédit (fig. 11), un tesson du haul de la panse d'une aiguiere rhodo-ionienne (fig. 12), i engobe blanc-crime et a décor de griflon dont il ne subsiste que á griffe sous une frise de languettes, sur un fond de rosaces el de svastika ${ }^{12}$; au-dessous, plusieurs fragments d'amphores et de sigillée (laire $1, B$ el. I) el fragments de céramique grise estampée.

Le sondage poussé jusqu'a 1.7\%) sous le sol de l'orchestre, a montré que la partie de ce secteur avail été remaniée au Bas-Empire : deux anses d'amphores étrusques, en pàte brun rougeàlre, haut de coupe ionienne a live à vernis noir. et fond d'une aiguière ionienne décolorée, céramique grise estampée a marli quilloché, et poterie luisante de l'époque de Constantin, dans un milieu de sable de plage et de coquillages marins a valves soudées d'eau saumatre (cardium edule) montrant qu'il ne s'acrit pas de déchets de ruisine.

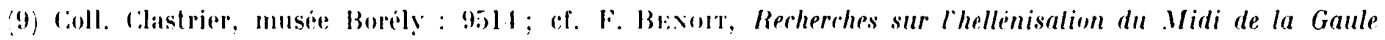
(196i), p. 1:1)

(10) Identifications de P. Mars: ces especces ont igalement eté signalees par H. RoLasw, l. r., p. 159. I ne "stratigraphie conchylohogique", faite dapris les fouilles du secteur de la plage Vivaux (V. XII), est exposé au

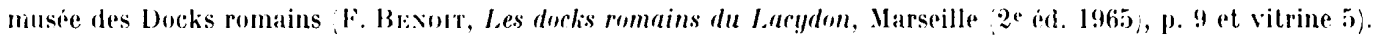

(1) Identification du Prof. lil. convernet.

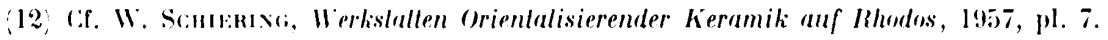




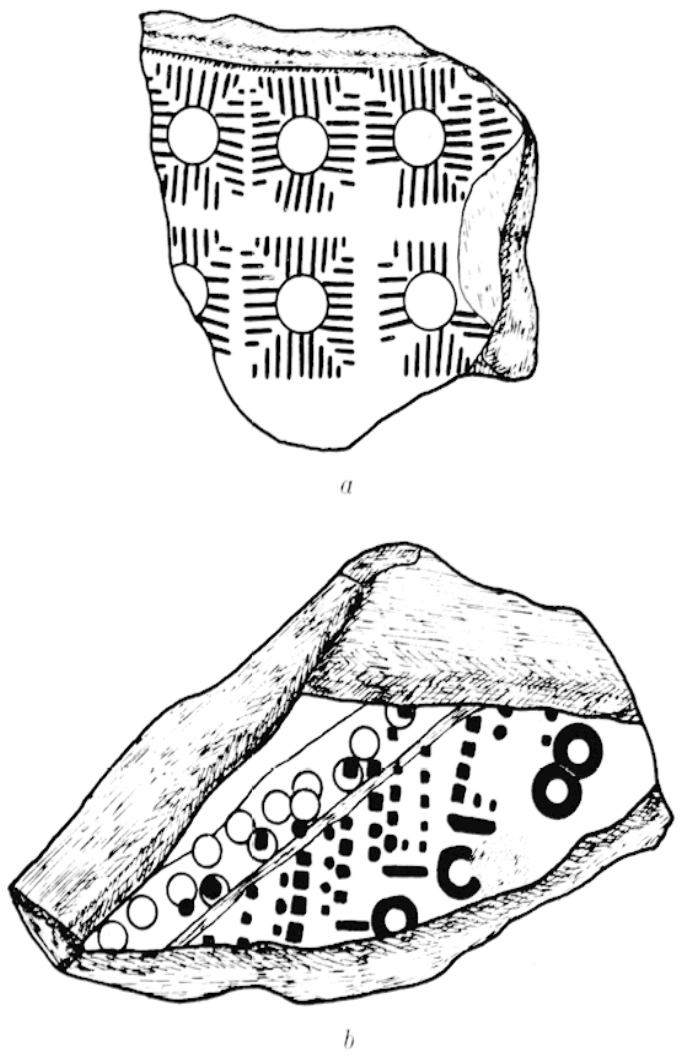

11. Tersons de ciramigue grise estampre.

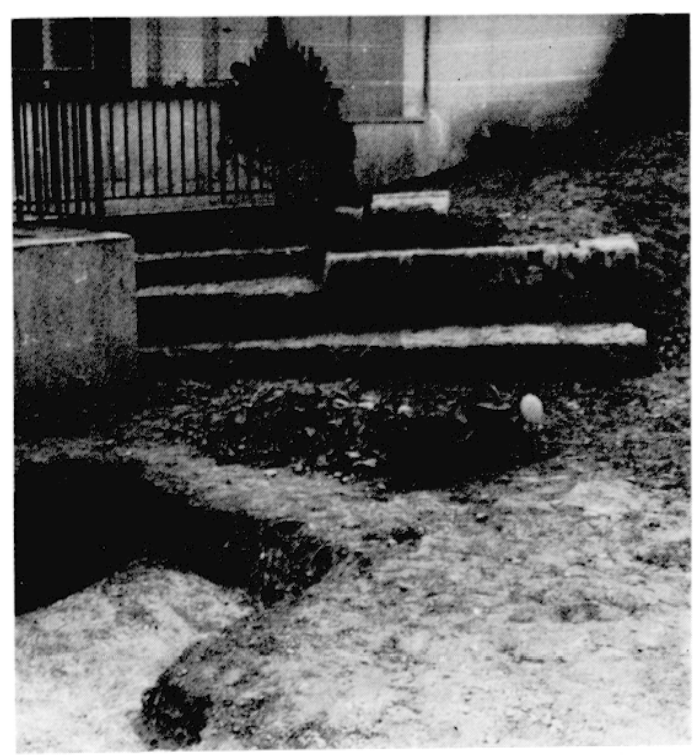

13. Mise an junr de la conche superienere du lit de dechets

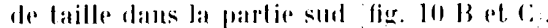

11. Coupe du remblat de l'orehestre, dans la partie sud - .

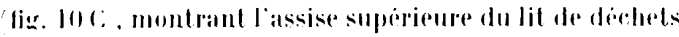
de laille et les couches altermeres de sable. de terre at de pierre.

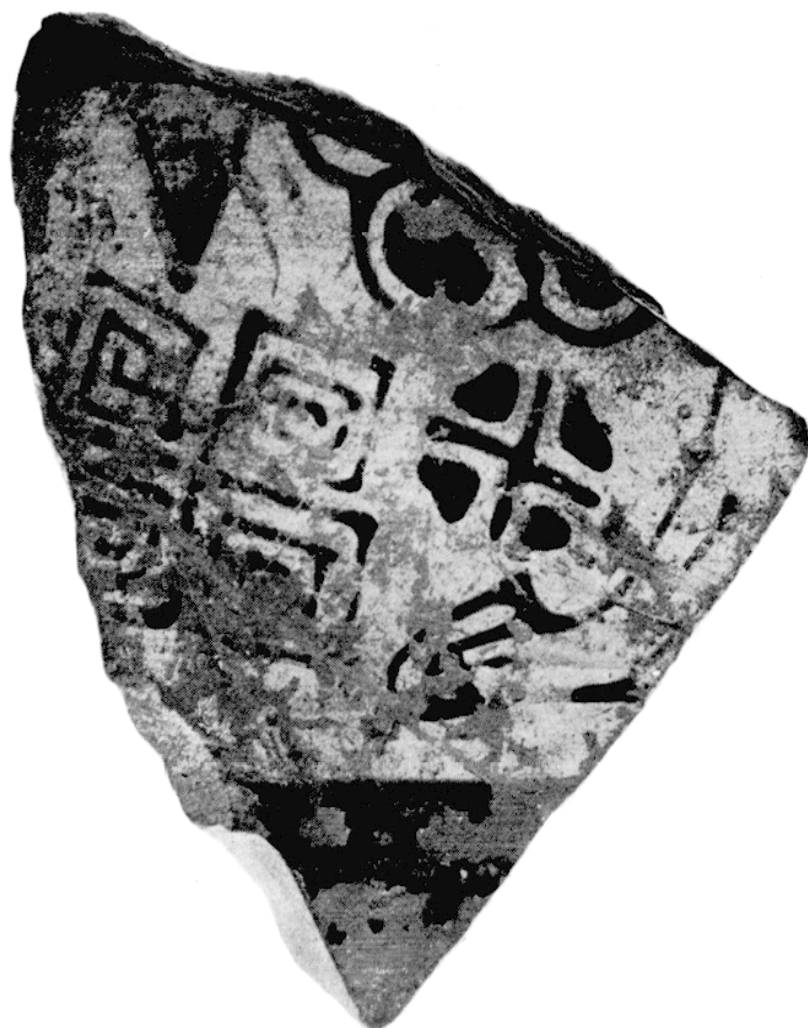

1:. Fragment diaguiare rhodo-ionienne an griffon.

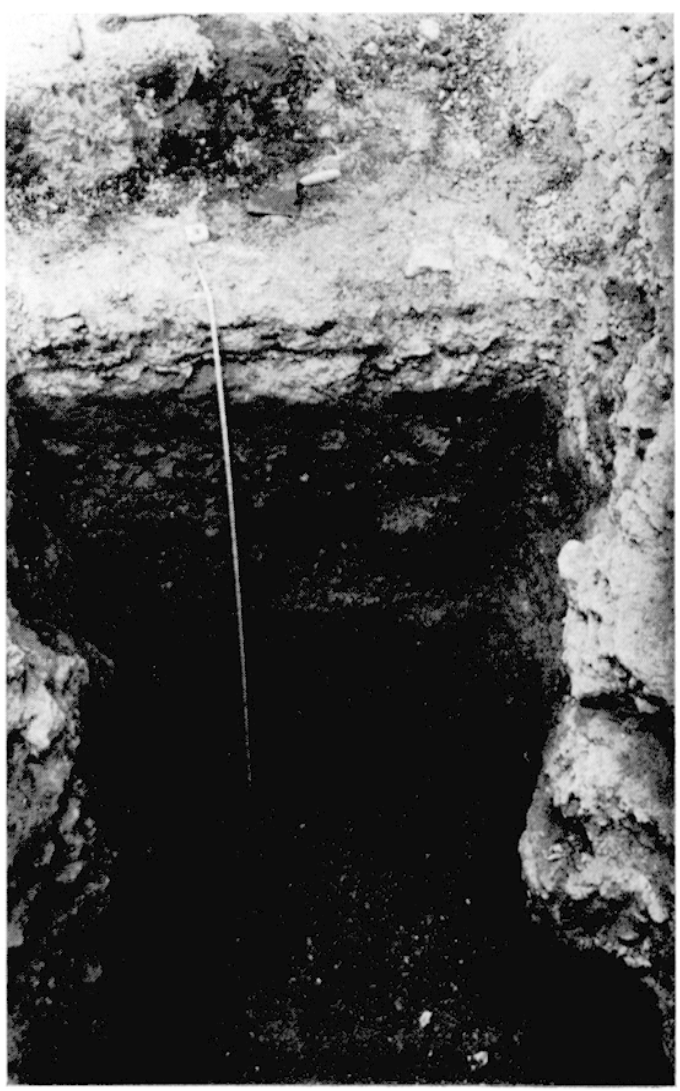




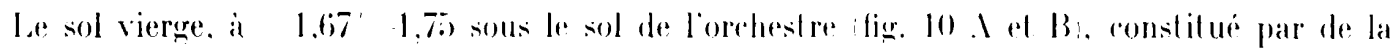
marne, conservait les I races de deux foyers aver charbon de bois. poterie indigine rouge micacé: au-dessus, deux P. B. tres corrodés (au droit : buste diademe : au revers: 2 Vicloires debout se faisant lace frappes a l'époque de Thédose Jer a Valentinion III. Ies apports de sable et coquillages rontimaient vers le n.-e.. ainsi que le montre la poursuite de la fouille en sape a ce niveau : six petiles monnaies de l'époque des lirandes Invasions donl une en argent aver robix. de bas til re. lriss colrodées. probablement frappées a Varseille. analogues a celles des Mliscampsos el du marlyrium de saint-Victor.

L'agrandissement de la fouille vers le nord a mis au jour la tranche du sol bélonne de l'orchestre (fig. 13) : elle a permis de faire un releve stratigraphique des diverses courhes de comblement. qui n’avaient pas été remaniées. Cependant. au niveau inférieur i lós). le enlissement de la rouche dapport marin eontenait encore deux P. B. analogues aux premiers. La couche de sable de plage. remplie de coquillages a valves adherentes. recouvant le sol vierore de marne. a donne une

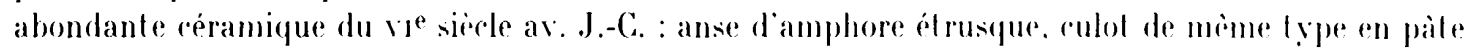
rouge usée par la mer. rebord de calice de Chios. delavé coupe ionienne a vernis noir ef a bande rouge intérieure, anse allique a glacure noire, rebord d'amphore massalible a levre replice parsemé de fines parcelles de mica : el des fragments romains auxquels elait adhérent le sable : rebord de

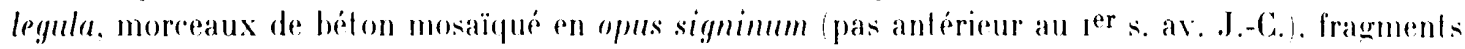
de panse d'amphores.

La poursuite du sondage vers le norel sous le lit superieur du beton de l'oreluestre (lig. 14), dans une partie qui etait invioleer a revele une stratigraphie analogur a celle des sondages de 1948, avere la meme inégalite d'épaisseur des rourhes de déchets de tajlle. de terre d'apport ot de sable, typique d'un comblement fait de main d'homme. Ies apports sont constitués par du sable de plage et de dune comprenant, avec des tessons ioniens uses par le roulis de la plage, un grand nombre de roquillages dont les valves claient encore soudées ensemble (fig. 10) (i). Sous le niveau de l'orehestre, un fragment de dalle de ralcaire de la Couronne de grande dimension fait partie du lit de déchets de taille; dans le lit supérieur de raillasse formant beton de l'orehestre, ont eté lrourés deux fragments de poterie a glacure rouge dont le vernis et la "fracture ondulante sont typiques d'Arezzo :

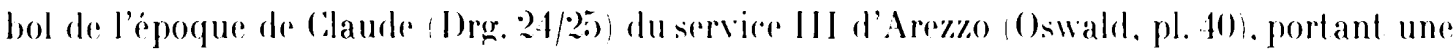
marque efface sur deux lignes : l: As/MA ?. hol a rebord droit guilloche avec gorge concave (type Ritterling i) du service II d'Arezzo) ot un fragment d'inseription en martore: ...R (?) ()..),

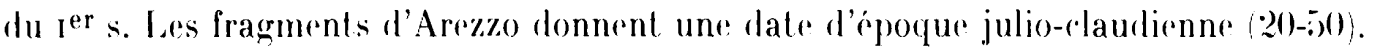

Il parait done que l'assise du théatre n'a pu ètre établie qu'à la suite de travaux considérables d'urbanisme, qui avaient pour fin de combler une dépression pour donner une assise a l'orehestre. dont la carea s'arlosiail a la butle. lin tel comblement n'a pu ritre fait qu'après la destruction de Marseille par César ; sa datation peut ètre précisée par la présence de fragments d'Arezzo d'époque julio-claudienne. er qui permet de reporter la construction à l'époque de Claude- Xéron. La constitution arlificielle de l'assise du théatre, à l'aide de remblais, montre qu'il n'avait pu sucéeder au théatre gres sur le mème cmplacement. Etant donne les courbes de niveau, il est vraisemblable que relui-ci, en bois comme le suppose Michel Clere, egalement arlosse a la butte saint-l aurent. fare a l'est. avait ete construit plus au nord à un niveau légerement supérieur, re qui avait entraìné sa dispa- 
rition. Il serait en ce (as dans l'axe du fortum de la ville hasse, etabli à la cote +3 m, au pied du mur de soulenement de la place de Lenche " $\left(\right.$ aves de saint-sauveur ${ }^{14}$ ), dont le dallage recenstruit au Bas-Empire avait conservé quelques dalles (musée Borély, XI, 2 et 3). son déplarement aurait eu pour rause l'extension du plan d'urbanisme romain, qui modifia ce quartier et régularisa le profil déenupe de la ralanque, par rétrécissement du plan d'eau du Lacyilon ${ }^{15}$.

Le nom mème d'agonolhèle. que portent deux inscriptions trouvées a Marseille at à .ice ${ }^{16}$. concernant les magristrats chargés de présider les jeux publies, et celui de cho-

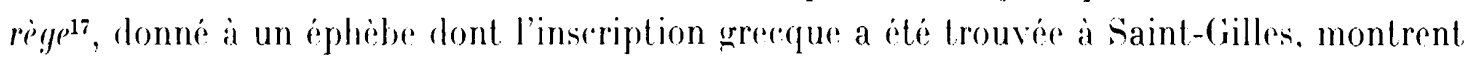
que cette institution avait une longue tradition remontant à l'époque de l'indépendance. On doit done conclure que le théâtre date du programme de reconstruction de la cité, après la destruction de César. Or. nous savons qu'un médecin célebre, d'origine marseillaise. Cirinas, descendant peut-ître de la famille de Pythéas, qui avait fait fortune a Rome par ses méthorles de médecine, sous Néron, avait légué à sa ville natale "dix millions de sesterces, apres avoir élevé les remparts de sa patrie ct d'autres constructions pour une somme à peine moindre $n^{18}$.

Michel clare, qui a fait la premiere élude sérieuse sur la topographie de Marseille, avait ete amene a dater de cette epoque les vestiges d'enerinte an grands blocs de pierre de la louronne qui avaient éte mis au jour en 1913, derriere la Bourse, place Jean-Giuin, aujourd'hui en loordure du cours Albert-ler. Le trace de cette enceinte, dans le terrain bas conquis sur le marais. ne paraissait pas en elfet correspondre au tracé oriental de la colonie greeque, d'apres la description du siegre de César. dont le ramp situe dans une position dominante. sur la butte des Carmes. commandait la butte des .loulins ol le rempart ${ }^{19}$.

Ine vérificalion s’imposait done.

\section{REMPART INE CIRINAS}

Il apparait que lo trace de cotte "nceinte est atteste par plusieurs autres élements. dont n'avait pas tenu romptr .I. Clere, an partirulier les deux assises en raleaire de la Couronne, comprenant huit bloce, tres corrodés, découverts rue sainte-Barbe, du coté de

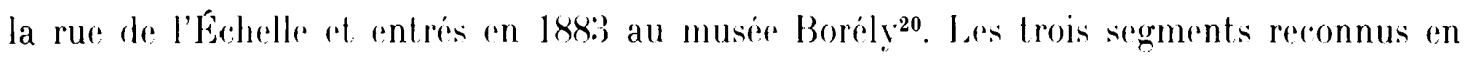
1913-1928. sont situés plus au sud, dans le terrain alors en cours de démolition, derrière la Bourse ancienne place dean-(iuin), aujourd'hui entre fe cours Albert-fer et la rue Bar-

(1.1) rit. infra, n. : $: 3$.

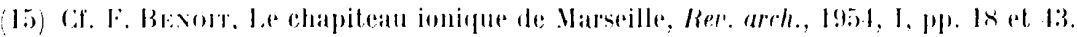

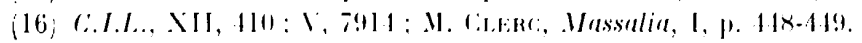

(17) $I .1 ; ., 2111$.

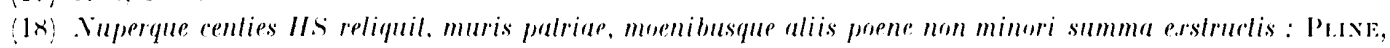

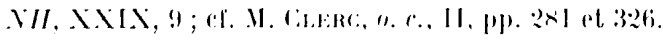

(19) .I. Ailks: II, p. I01.

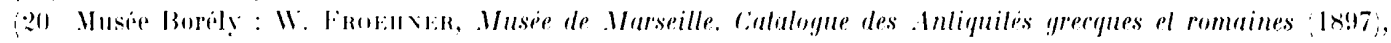

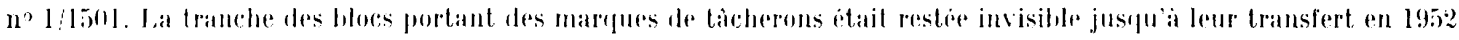

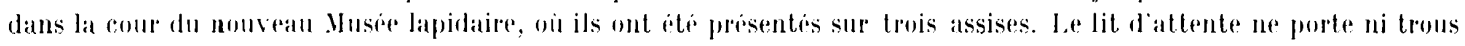
de lomve pour lo levage, ni mortaises pour le secllement de gonjons. 


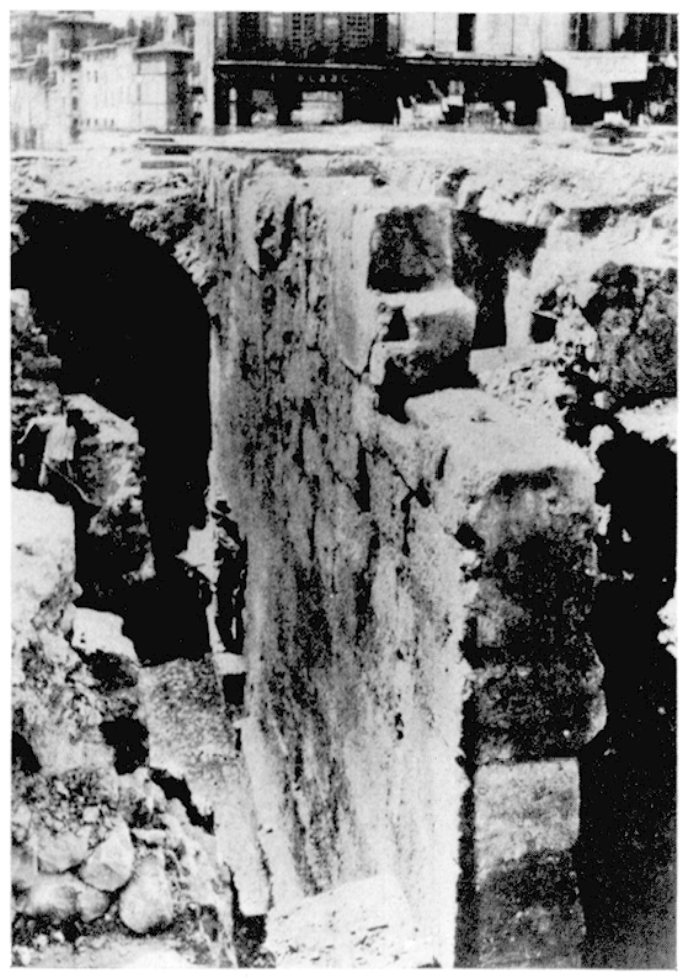

15. Enceinte. Partie de mur conservée dans une cave de la place Jean-(iuin, montrant la coupe du parement (1916).

busse. L'un d'rux, rlassé monument historique Ir e.2. déembre 1916, long de $16 \mathrm{~m}$, comprenant huit assises (servant de fondation i la facade d'un immeuble) (figr. 15), a elé retrouré en 1959. dans le ravau ou il arail ofie (onservín.

Les uns ot less autres, en appareil rectangulaire isodome. datent de la mème époque; trois des premiers hlocs portent sur la tranche la mème marque, AP lies, que deux des boutisses du mur de la place Jean-fiuin.

A première vue, ce rempart paraissait donc remonter à l'épocque greceque, par la presence de signes grees et par sa similitude l'appareil aver celui de Saint-Blaise, du ${ }_{\text {III }}{ }^{\mathrm{e}}$ siècle avant notre ère, et avec les constructions de (ilanum ${ }^{22}$. La discussion fut d'autant plus vive que le calcaire de la Couronne était encore employé au xvir siècle et qu'un autre édifice, les "liaves de SaintSauveur", servant de soutènement à l'abhaye de religieuses reconstruite en 1689 et fondé au niveau du dallage du forum du Bas-Empire (fouille XI), avait un parement, sans boutisse, d'appareil analogue, reconnu sur onze assises (haut. des blocs 0,40 à 0,50$)^{23}$. L'appareil également isodome est a joints vifs, mais sans le chanfrein des arêtes qui caractérise le mur de la place Jean-Guin.

(i. Vasseur mentionne que l'épaisseur du mur varie de 0.75 ia 0,90 el que les deux faces en sont dissemblables : celle de l'ouest est tress irréguliere, avec des pierres plus ou moins saillantes (fig. 15), tandis que la face extérieure vers l'est (la seule visible aujourd'hui) est d'une exécution tris soignée. (Observation qui montre qu'il ne subsistait que le parement du mur. dont le blocage

(21) Sur la "redecouverte" de ce monument historique: Inventaire des fouilles, XXX et II.-P. Lyor'x, IIommes el dieux de la Gaule 1961$)$, p. 27.

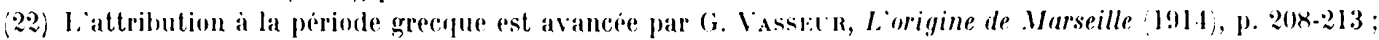

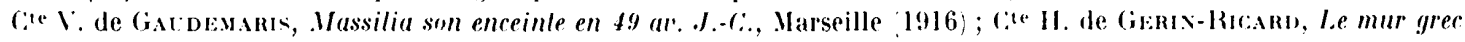

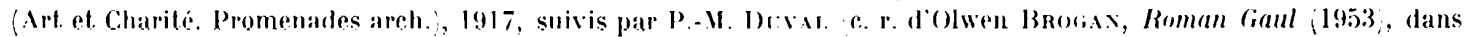

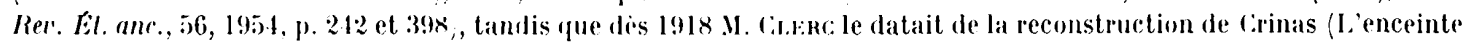
grecque de .larseille, Rer. El. anciennes, :0, 1916, p. 47 el Massalia, 1I, 1929, p. 286, et qu'L. I) prat le rajeunissait

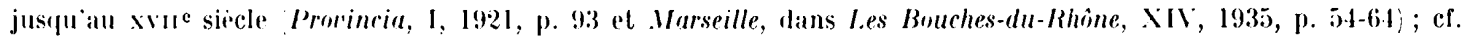

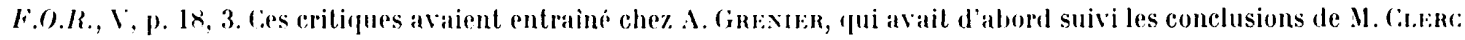
(Man. Arch. gallo-rumaine, I. 1931, p. 2866', un certain doute sur l'authenticiti de cette enceinte : Topographie marseillaise, Rer. Eludes anciennes, 36, 1934, p. 390.

223, Plan de 1773 dans J.-B. Cirossos, Rerueil des anliquiles el monumens marseillois :1773, p. 22:3 et pl. 36 ;

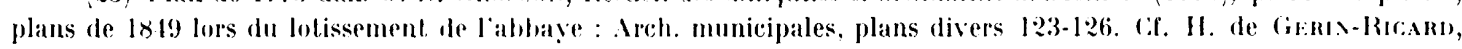

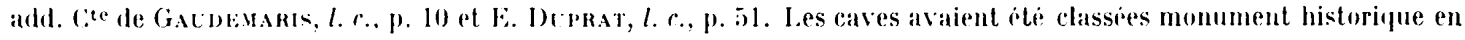
1810. lin 1953, le projet de fouille fut suspendu et les caves furent recousertes par le Service des Ponts et chaussies d'une voùte en beton avec porte "fictive". 

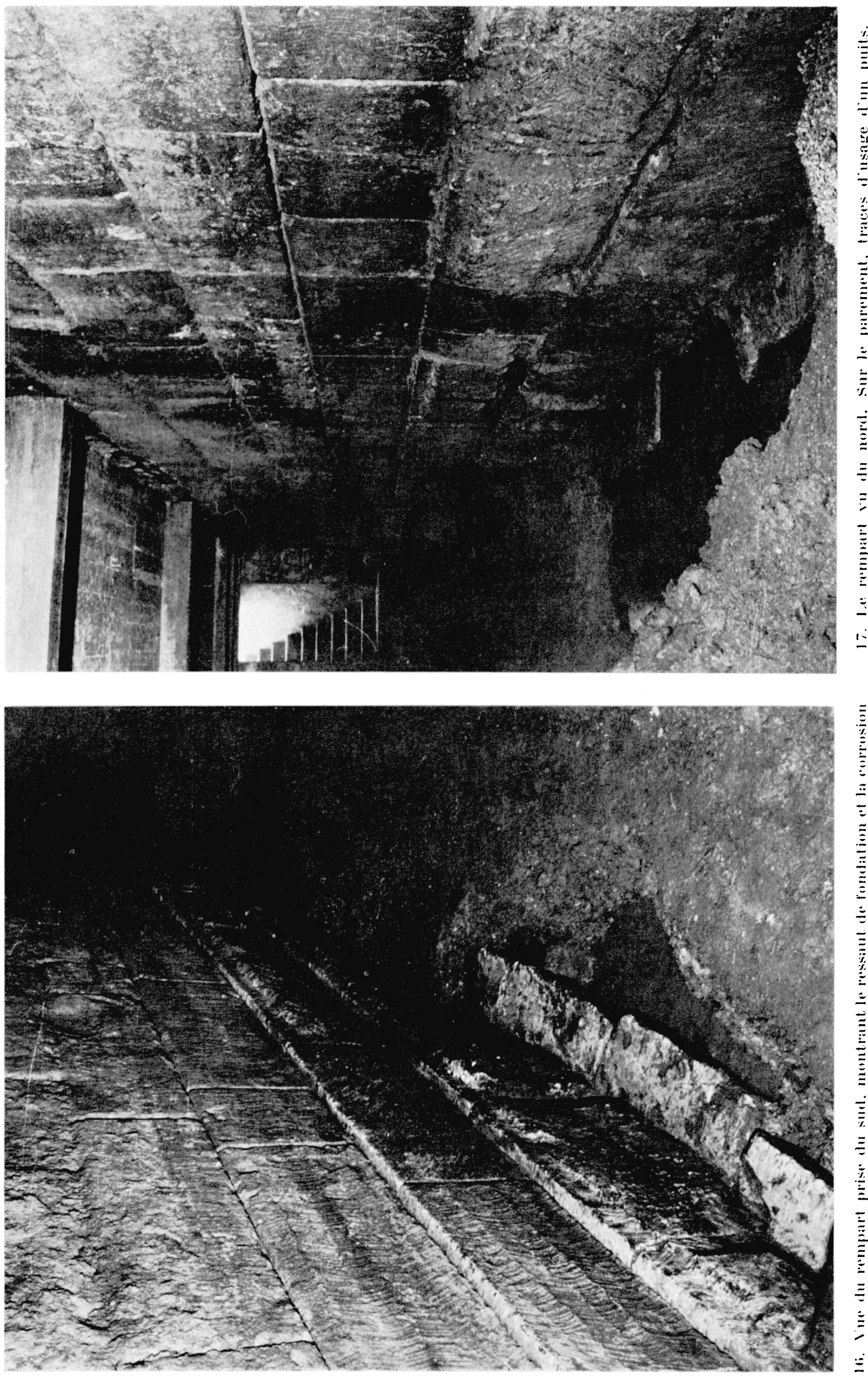
interieur avait disparu. lors de la réutilisation de ce mur comme facade d'un immeuble. les coupes faites par II. (jérin-Ricard el reproduites par E. I Uuprat ${ }^{24}$ notent onze et treize assises en place, dont les trois (une fois quatre inférieures sont en retrait l'une sur l'autre. l'assise de fondation étant, largement empattée (coupe de la fig. 2:3. Ce profil rorrespond a relui du segment de mur retrouvé. Cependant les dimensions données aux blors varient entre 0.42 el 0.57. alors que reux-ci ont uniformément 0.50. a lexception de la seconde assise de base portant les marques de laicheron sur la tranche des boutisses. qui alleint 0.62 . Vais la mème irrégularite de hauleur se retrouve dans les blocs de la rue sainte-Barbe variant de 0.50 i (0.57. leur epaisseur de 0.j.l en moyenne alleignant 0.7x dans un bloc. Celle inécalite est le premier caraclere qui dillérencie ce rempart de celui de saint-Blaise. dont les blocs ont une hauteur uniforme de 0.4547.

In sondage au pied du mur. en vue de reconnaitre la technique de sa construction, a montré que le ressaut de la derniere dalle correspondait au ressaul de fondation (fig. lib établi sur un sol raillouteux envahi par les eaux d’infiltration et ayant ronserve la trace d'un puits lig. 17 s. sans doule destine a donner une assise plus compacte au terain vaseux de celle ancienne anse du largdon, reconmu 100 m a l'est dans le sondage du Cours Belsuncees. Les deux assises qui reposent sur celle dalle de fondation sont elles-mèmes en léger retrait l'une sur lautre ce qui permel de supposer que le sol antique rorrespond a la $3^{2}$ assise. (On ne peul se fonder sur les observalions pseudo-scientifiques de ledoyen. dont la straligraphie. faile dans des terres de comblement. a elo justement reriliquées par li. Inupral.

La construction est done toute differente de celle de l'enereinte de saint-Blaise. qui repose sur l'assise du hane de rorhere En ellot, ce mur repose sur a une solide semelle dont

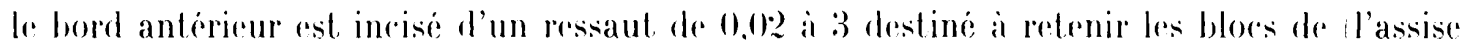
superieure) ". La coupe du mur de saint-Blaise montre que rette dalle a ressaut. qui indique le niveau du sol, est elle-meme supporté par b) ou 6 assises de fondation, reposant sur le rocher. II. Clere qui assista a la mise au jour du mur de la place Jean-Guin en 1913 ret. 19:-8 at qui data, le premier, le mur de l'époque dr Jéron, avait noté que les bloces avaient une bordure risclíe (anathyrose), selon la tradition greceque, raractéristique du mur de Saint-Blaise alors incommu, que l'on retrouve egalement dans les gradins du théatre de

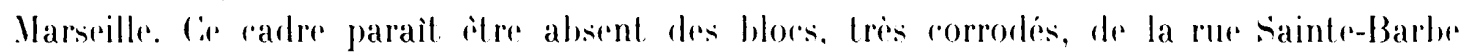
(fig. 2). Il avait en outre remarque que la plupart de ces blocs ctaient lies entre cux, au milieu de leur epaisseur. jar une mortaise en double queue d'aronde. entaillé sur le lit

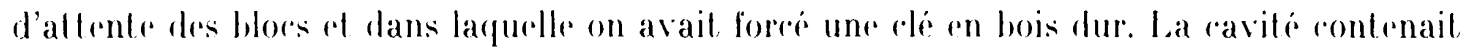
parfois eneore une couche de poussiere lignense el jaunatre prosenant de la decomposilion

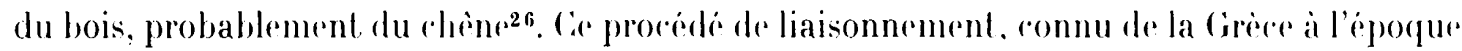
archä̈que, a cte observé au "mur des Païens" a sainte-()dile. pres de strasbourg ot a la Frankenhureg pres de sichlestadt², mais est inconnu a saint-Blaise et dans le serment de: mur de la rue Sainte-Barlee (ligr. 18).

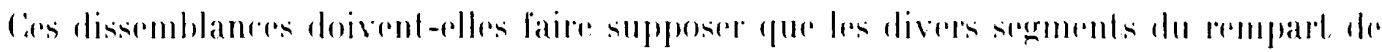

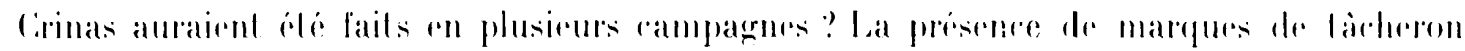

24, Marseille, l. r.. p. 5!). tig. 1-6.

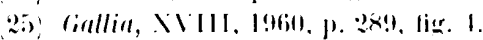

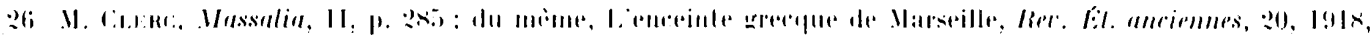

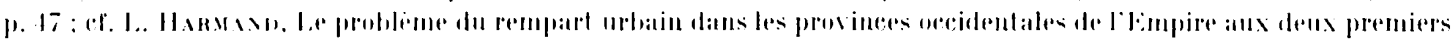

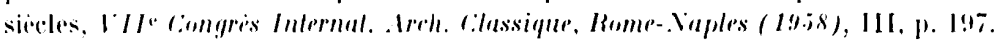

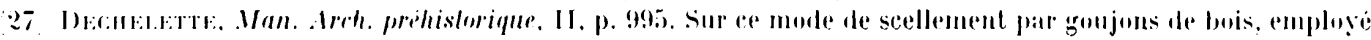

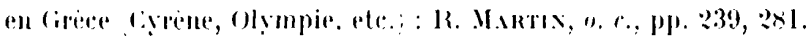




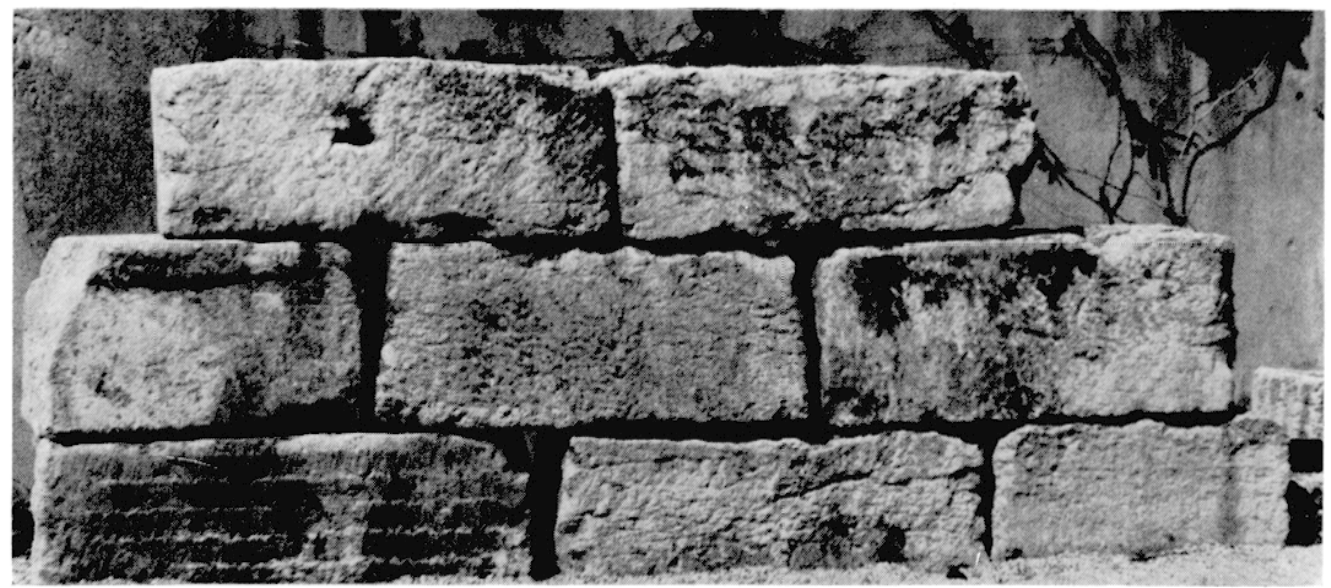

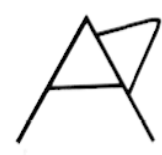

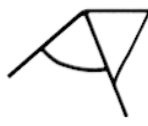

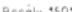
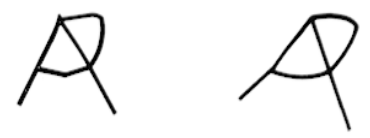

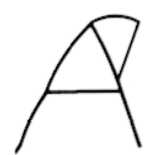

1x. Fiments du rempart de la rue Sainte-Barbe, au muse Boriely (1952).

* 19. Releve des marques de tacheron du rempart de la rue Sainte-Barbe (musce Borély la(l) et de la place Jean-ciuin Cours Allort ler).

greecques communes aux deux segments du rempart et identiques par leur graphie (fig. 19), montre qu'il n'en est rien, tout en écartant un rapprochement avec ceux de Saint-Blaise.

I)rux des boutisses du mur de la place Jean-(Guin portent la marque AP liés (fig. :20); la mime marque se retrouve sur la tranche de trois blocs de la rue Sainte-Barbe imusée Borély), ayant sans doute servi également de boutisses, re qui expliquerait la conservation sur l'un de ces blocs de la taille en arètes de poisson (ligr. 21). De tels signes ne peuvent être confondus avec la marque du chantier sur des "pierres de libage" des carrières de la Couronne, d'époque moderne ${ }^{28}$. L'archaïsme du rô à boucle brisée, qui contraste avec la forme gréco-romaine de l'alpha, ainsi que le remarque. I. Clere pour les deux premiers signes, a pour cause la difficulté que présentait pour le lapicide la gravure sur pierre et ne pourrait itre, au plus, antérieur à 150 av. J.-(..29. Ces signes ne se rencontrent pas dans le répertoire de Saint-Blaise el correspondent à des marques de tâcheron sur le chantier des carrières de la Couronne et non à des indications de pose ou à la numérotation des blocs, romme le ras se présente a Saint-Blaise. Sans doute AP sont-elles les initiales du nom du maitre de chantire, qui avait ronservé son nom hellénique et l'usage de l'écriture greecque: Aristion, Aristocles, etc.

Seule est apparente. nous l'avons dit, la face extérieure du mur, vers l'est. La vérification des observations de Vasseur at de Clere ne pourra done itre tenté que lorsque sera

28) F. I)renat, Marseille, l. c., p. 57.

29) .I. II.:Rc, J Ienceinte grecque de Marseille, l. c., p. 50 ; Massalia, II, p. 286. 


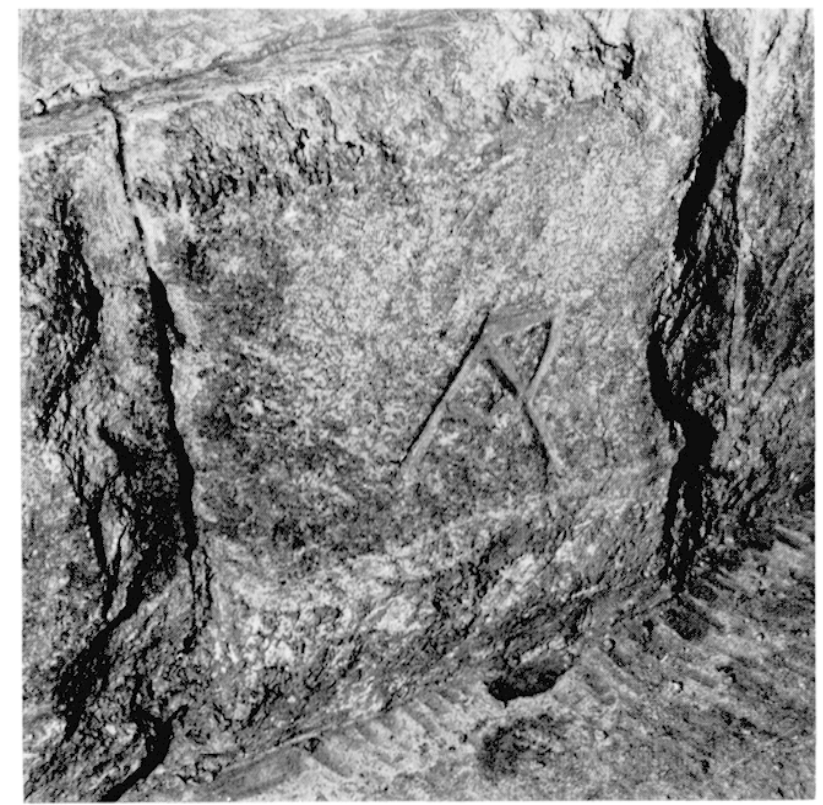

20. Marque de lacheron sur butisse du rempart place Jean-cinin

21. Marpues de tacheron sur la tranehe des houtisses du rempart me sainte-Barter.

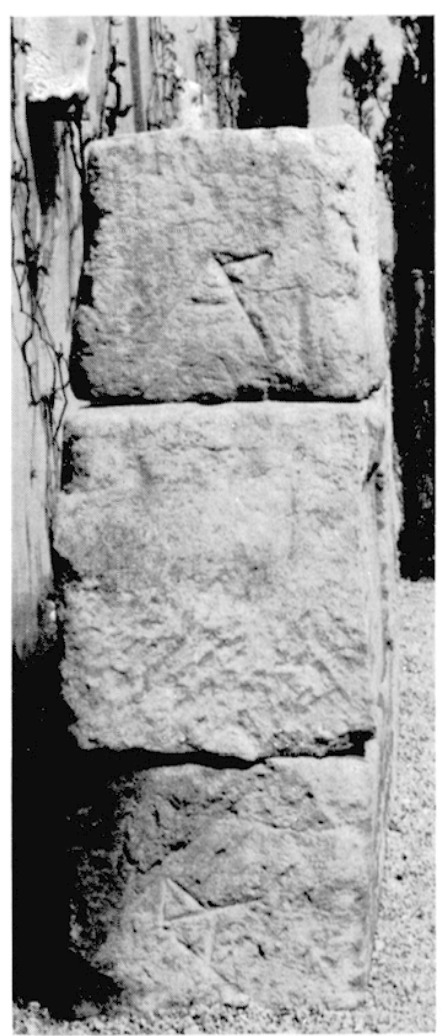

faite la coupe du mur, au moment de l'ouverture du chantier de construetion, qui doil le sauvegarder.

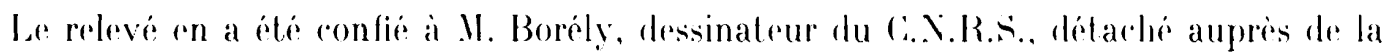
l)irection (fig. 2:3). L'appareillage est a joints vifs comme a saint-Blaise el présente la mème taille de stries diagonales, dites uen arètes de poisson ". Vais le ravalement dees blocs a Saint-Blaise avait ete obtenu apres la pose. en cernant les quatere cotes du blor d'un listel de 0.02/3 de largeur. tandis qu'a Varseille. les arètes des boes sont chanfreinéres, a fin d'éviter d'épaufer les arrites au cours de la pose. Cette technique. inconnue a saintBlaise, a été signalée en Cirèce, notamment a Thasos ${ }^{30}$. La farture "'n arètes de poisson" est également différente : "les ravalements avaient été obtenus a saint-Blaise à l'aide d'un outil tranchant qui a laissé sur la pierre des traces légerementent concaves, disposées en longues lignes de chevrons courrant la face des parpaings. alors que le ravalement en place des joints a cerné le bloc d'un listel de 2 a a 3 c'm de largeur "31. Le mur de Marseille présente par contere des striures a tracé biseauté fig. 16 ot 18 . faites avant la pose an martean i double taillant (laye). La laille en arètes de poisson n'est visible que dans les assises inférieures, dont la limite donne la hauteur du comblement qui les a protegées depuis le llaut Moyen Age. Cette taille, tres differente de celle du mur de Saint-Blaise, l'rst exalement de relle du mur des "Caves de saint-saureur".

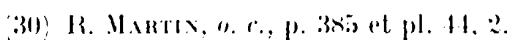

(31) H. Roldaxn, Fonilles de trant-Blaise, 1 1951), p. 91. 


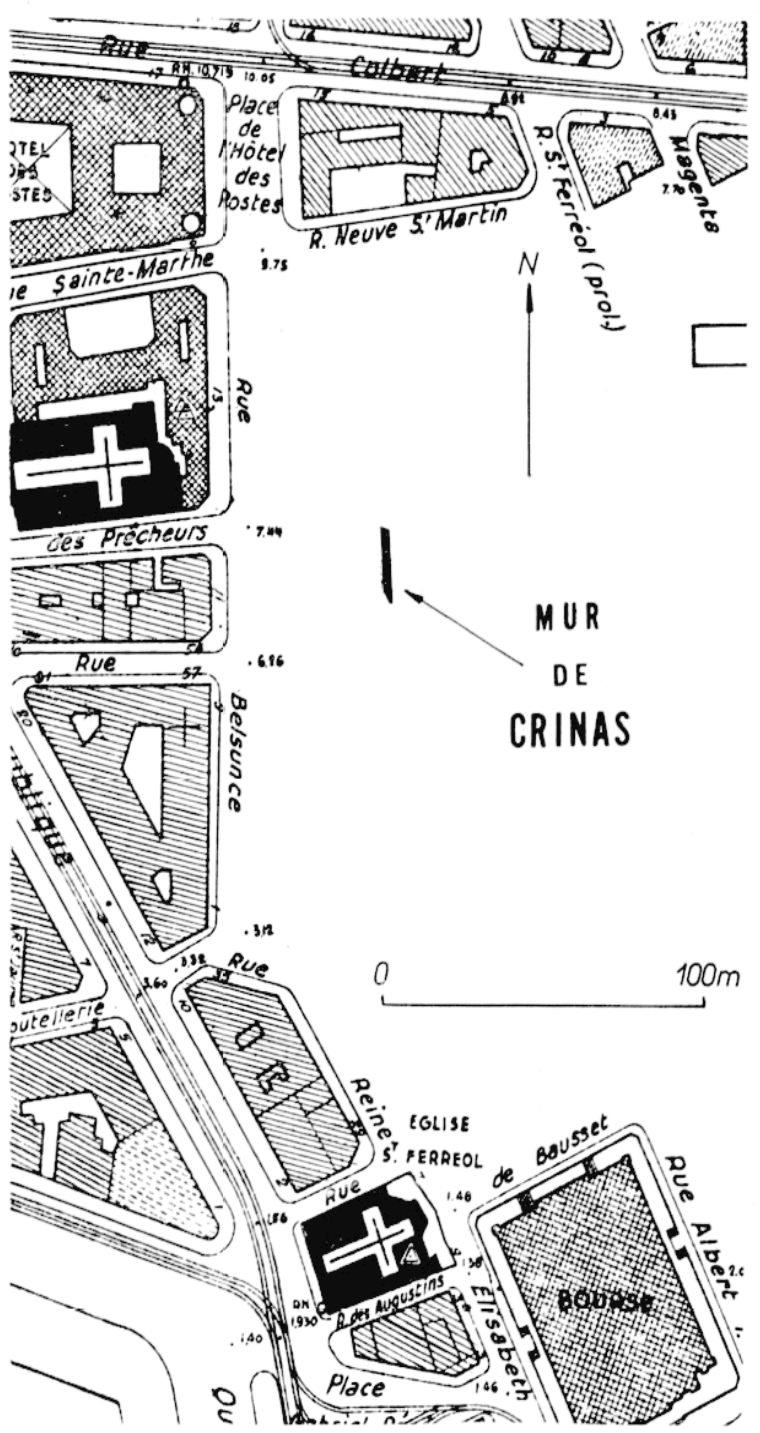

22. Plan de situation du mur de lininas.

Le rempart de Cirinas et celui de Saint-Blaise obéissent done à une même tradition arehitectonique, mais leur terhnique diffère profondément. Il est intéressant deconstater avec H. Rolland que celle tradition "héréditaire" avait persisté dans les constructions en grand appareil de Gilanum, dont le péribole du sanctuaire de source. a porte en chicane et merlons arrondis, paraît être inspiré du mème modèle que l'architecture militaire de Saint-Blaise ${ }^{32}$. La ressemhanere ast plus frappante encore dans la Leihnique des substructions en grand apparril qui soutiennent la Maison de sylla (3:) av. J.-(i.) at le péribole des temples jumeaux élevés sous Agrippa : par le nombre de leurs assises, elles sont plus proches de l'arehilecture de saintBlaise que de Marseille. si l'on date les monuments de Glanum antérieurs à l'Empire du $\mathrm{I}^{\mathrm{er}}$ siècle avant J.-C., on ne jeut qu'itre frappé de la dégénérescence de la lerehnicque greerque dans le monument marseillais, attribué à l'époque de Néron.

Il ressort de cers observations que ce rempart, tant par sa situation que par sa ronstruction, n'était pas un vrai mur de défonse, mais un rempart élevé ad pempram el oskenlalionem. qui rendait a Vlarseille un reflet de son ancienne grandeur. Il délimite la ville reconstruite à l'epoque romaine, dont la superficie ne paraît guère ètre supérieure a 32 heetares ${ }^{33}$. Ainsi que l'avait reronnu .V. Clere, se fondant sur la topographie de la ville lors du siegre de César, il doil etre attribué au programme de reconstruction dù

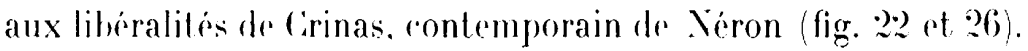

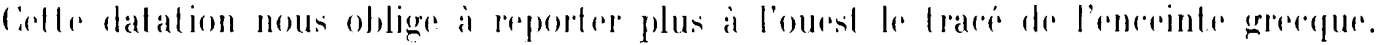

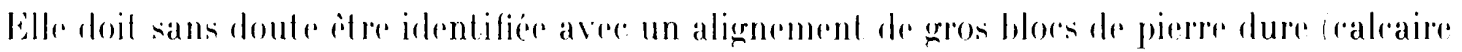

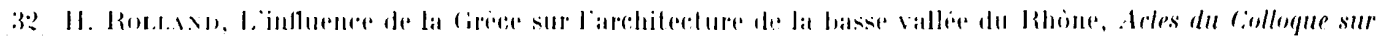
les influences helleniques en Gaule Publ. I niversiti de D)ijon, 1957, p. 119 .

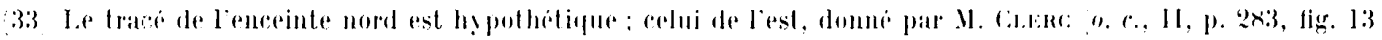

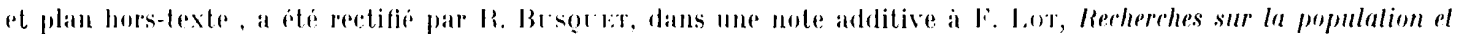

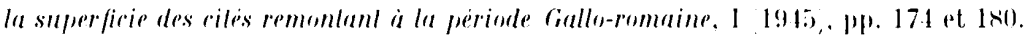




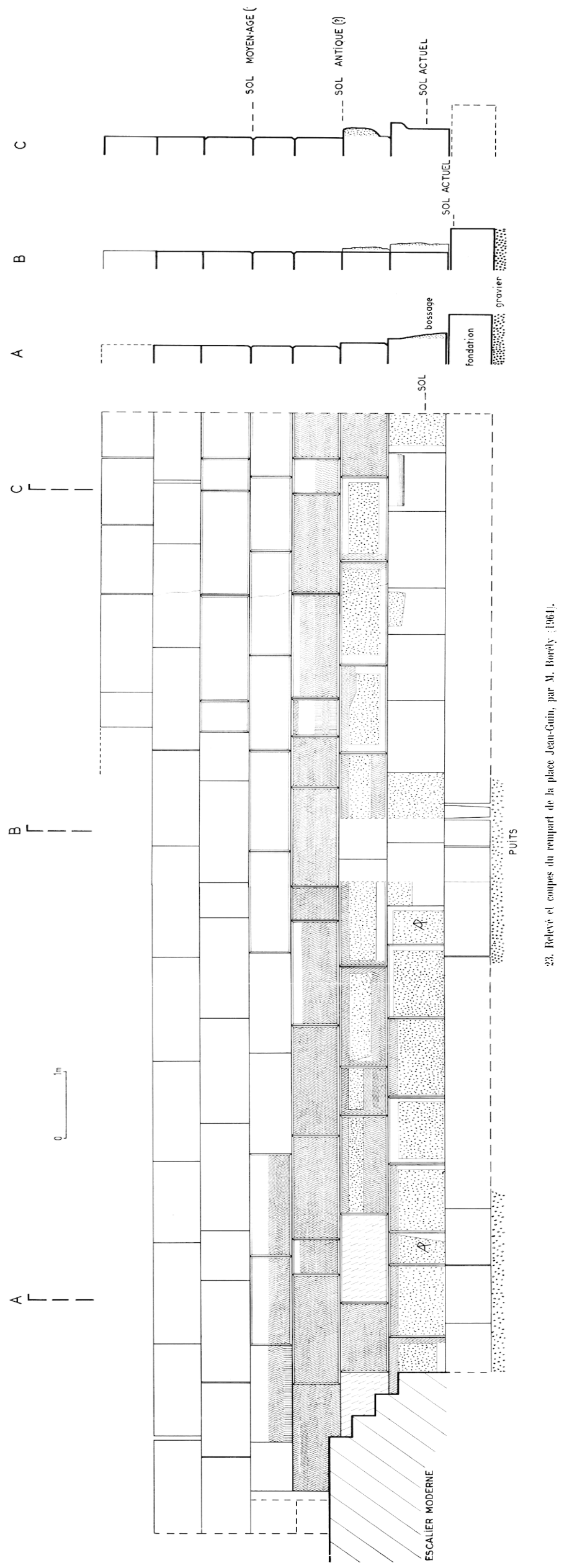


oligorene mis au jour rue Xégrel. à mi-pente de la déclivité de la colline. entre el sous les caves des maisons bordant rette rue a l'est. Rasée a l'époque romaine, elle avait retenu un tress important dépotoir dans lequel avaient été trouvées en 18633 les stiles d'Artémis archaïque "t dont la fouille a fourni une abondante réramique, du ${ }^{\mathrm{e}} \mathrm{e}^{\mathrm{e}}$ siècle à l'époque romaine fouilles rue .ergrel l). Ceet alignement de hlocs qui n'etail qu'une assise de base. fondée sur le poudingue, gravissait la colline en gradins. Il a éte préleve un élement du parement, d'une longueur de $2.10 \mathrm{~m}$ ifig. 24 , aver son horage interne conserve sur $1.20 \mathrm{~m}$, ce qui donnait une épaisseur de $1.60 \mathrm{~m}$ emviron, sans le parement interne. qui avait disparu ${ }^{34}$.

Sans doute soutenait-il un mur en briques d'argile crue, dont la terdenique, signalée dans l'oppidum des Pennes ot l'habitat de Ilouriess s'etait maintenue sous l'Empire. ainsi que le montrent less vestigess retrouvés dans les ruines des dorkis romains du largdon, datant de l'époque des Flaviens. L'assise du rempart avait subi des restaurations. qu'atteste la presence d'un Ci.B. Lress corrodé de Marseille au taureau, date du HI $^{\circ}$ siede. lans la rouche supérieure du blocage. Élahli a mi-pente de la buttr des Moulins il'arropole de la rite? ). il itait separé par une dipression marécageuse, vers le surl, le la butle

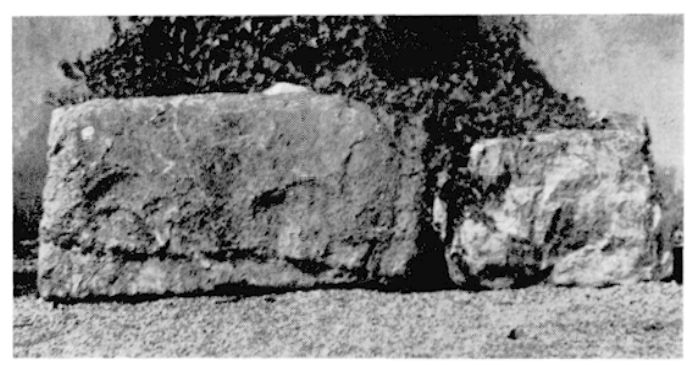

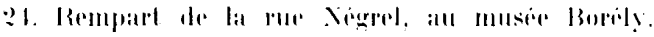
des ('armes, où César aurait elabli son camp. Son tracé represente done une notahle extension de la cite vers l'est et le nord-est, le

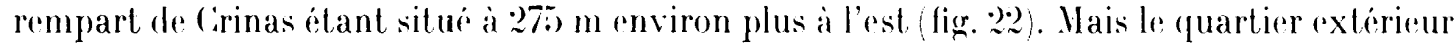
a ce rempart était habité des l'époque greeque ct formait un faubourge. que l'on doit

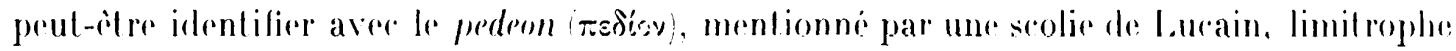
du rempart à l'époque du siege de Cósar, press des . Varalia ${ }^{35}$.

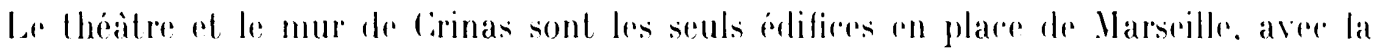
portion des dorks romains découverts en 1947-1836 al aménagés an 19663) an "musée de siten et le marlyrium rupestre du II e$^{e}-\mathrm{v}^{\mathrm{e}}$ siècle reconnu sous le sol de la chapelle ColreDame-de-Confession a l'ahbaye saint-Virtor ${ }^{37}$. La cité enrecque comptait rependant de grands monuments, dont le seul témoin est te chapiteau ionique en rateaire de la couronne, retrouvé en remploi dans le quai romain, à la pointe de la butte saint-laurent. datant du

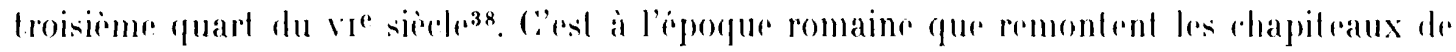

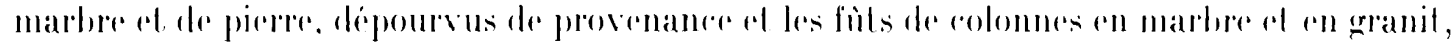
fig. 1.

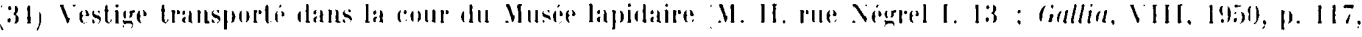

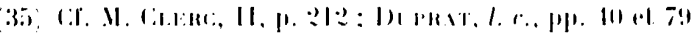

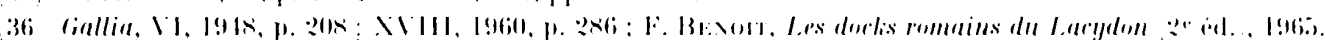

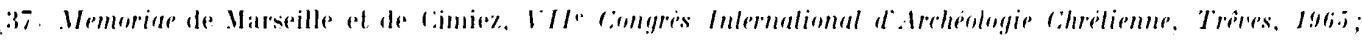

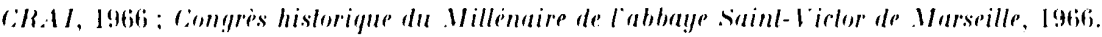

38. F. Bexort, le chapitean ionicue de Marseille, l. r. 
remployés dans la construction dr l'alrium de l'ahbare Saint-Victor, au ve siècle, ainsi que les rares fragments arehitecturaux en ralcaire de la louronne, trouvés sans contexte:

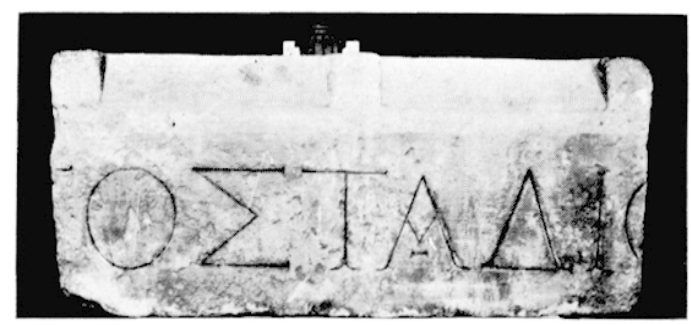

25. Fliment de stỵlohate du stade. all musce Boroly.

fragment de frise (long. 0.6i): haut. (0.5) ; ep. 0.35). du aer s. tres corrodé, remploye au $\mathrm{xI}^{\mathrm{e}}$ siecle dans la reconstruction de l'abbaye saint-Victor ${ }^{39}$ :

émemen de sylobale du stade (long. 1.42: haul. 0.57: ep. 0.40) Iromé dans la dimolilion de la Vieille Major el portant linscription grecoue:

\section{T $0 \sum \mathrm{T} .1 \Delta \mathrm{I} 0 \times 40$}

Remployé a l'époque chrétienne, et transforme en couvercle de sarcophage en batiere orné de six acrotieres (fig. 25) aver une croix gravée au centre, il provient du stade. qui devait âlre adosse a la butle sur laquelle s'éleve la cathédrale peut-ìtre a l'exlérieur de l'enceinte. son épigraphie est d'époque romaine. Doil-on le faire descendre jusqu'au ne siècle. comme le suppose .I. Clerc? si lon remarque que ses tranches latérales ont un cadre ciselé. comme les gradins du théal re el que sa hauleur est analogue a relle de certains éléments du mur de Crinas. on esl amené a l'allribuer également a la reconstruction de la ville sous Veron (tig. 26).

26. 1 theatre

2 mur de Crinas

3 mur rue Négrel

4 docks

5 forum

6 enceinte nord

7 littoral grec

8 littoral romain

9 nécropole rue Tapis Vert

10 navalia

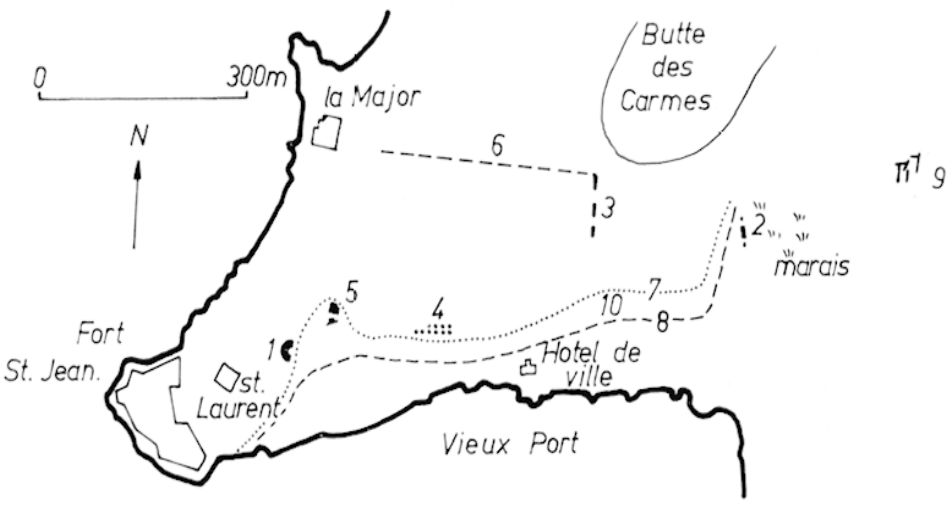

Ces edilies montrent que l'hellénisation de Marseille avail surveru a la perte de son indépendance at nous explique le "reflet hellénique " des monuments grallo-romains de Provence, d'Aples, du Vernègues, dre Gilanum, - qu'a si bien note Miehel lilere : "C"est l'art srere qui survil sous l'art romain $\|^{\text {th }}$.

Firnand Brisort.

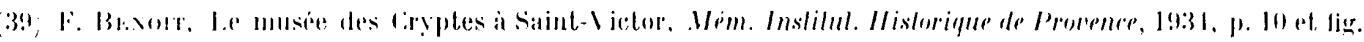

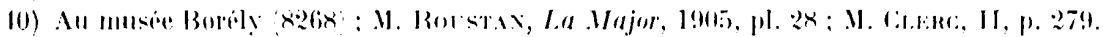

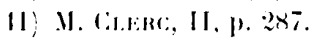

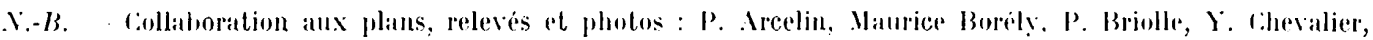

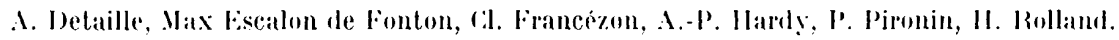

